\title{
On reverse-order law of tensors and its application to additive results on Moore-Penrose inverse
}

\author{
Krushnachandra Panigrahy ${ }^{1} \cdot$ Debasisha Mishra $^{1}$ (D) \\ Received: 28 April 2020 / Accepted: 3 August 2020 / Published online: 12 August 2020 \\ (c) The Royal Academy of Sciences, Madrid 2020
}

\begin{abstract}
The equality $\left(\mathcal{A} *_{N} \mathcal{B}\right)^{\dagger}=\mathcal{B}^{\dagger} *_{N} \mathcal{A}^{\dagger}$ for any two complex tensors $\mathcal{A}$ and $\mathcal{B}$ of arbitrary order, is called as the reverse-order law for the Moore-Penrose inverse of arbitrary order tensors via the Einstein product. Panigrahy et al. [Linear Multilinear Algebra; 68 (2020), 246-264.] obtained several necessary and sufficient conditions to hold the reverse-order law for the MoorePenrose inverse of even-order tensors via the Einstein product, very recently. This notion is revisited here among other results. In this context, we present several new characterizations of the reverse-order law of arbitrary order tensors via the same product. More importantly, we illustrate a result on the Moore-Penrose inverse of a sum of two tensors as an application of the reverse-order law which leaves an open problem. We recall the definition of the Frobenius norm and the spectral norm to illustrate a result for finding the additive perturbation bounds of the Moore-Penrose inverse under the Frobenius norm. We conclude our paper with the introduction of the notion of sub-proper splitting for tensors which may help to find an iterative solution of a tensor multilinear system.
\end{abstract}

Keywords Tensor · Moore-Penrose inverse · Einstein product · Reverse-order law · Perturbation bound $\cdot$ Sub-proper splitting

Mathematics Subject Classification 15A69 $\cdot$ 15A09

\section{Introduction}

Many phenomena are modeled as multilinear systems in engineering and science such as isotropic and anisotropic elasticity are modeled [16] as multilinear systems in continuum physics and engineering. Tensor methods have been used to solve problems in quantum chemistry: the fundamental Hatree-Fock equation is solved by Khoromskij, Khoromskaia, and Flad [13] and the works on multidimensional operators in quantum models were done by

Debasisha Mishra

dmishra@nitrr.ac.in

Krushnachandra Panigrahy

kcp.224@gmail.com

1 Department of Mathematics, National Institute of Technology Raipur, Raipur, Chhattisgarh, India 
Beylkin and Mohlenkamp [2,3]. Multidimensional boundary and eigenvalue problems are solved by Hackbusch and Khoromskij [7], and Hackbusch, Khoromskij and Tyrtyshnikov [8] through separated representation and hierarchical Kronecker tensor from the underlying high spatial dimensions using a reduced low-dimensional tensor-product space. A tensor is a multidimensional array. An Nth-order tensor is an element of $\mathbb{C}^{I_{1} \times \cdots \times I_{N}}$ which is the set of order $N$ complex tensors. Here $I_{1}, I_{2}, \ldots, I_{N}$ are dimensions of the first, second, $\cdots, N$ th way, respectively. The order of a tensor is the number of dimensions. A zero-order tensor is a scalar, a first-order tensor is a vector while a second-order tensor is a matrix. Higher-order tensors are tensors of order three or higher. These are denoted by calligraphic letters like $\mathcal{A}$. $a_{i j k}$ denotes an $(i, j, k)$ th element of a third order tensor $\mathcal{A}$, and in general, $a_{i_{1} \cdots i_{N}}$ represents an $\left(i_{1}, \ldots, i_{N}\right)$ th element of an $N$ th order tensor $\mathcal{A}$. For more details, we refer to the recent books $[5,23,24,30]$ on tensors.

There has been active research on tensors for the past four decades. For applications and tensor-based methods, we refer the readers to the survey papers $[14,15,17]$ and the references cited therein. But, research contributions on the theory and applications of generalized inverses of tensors are very little. The first work in this direction was reported in 2016 (see the article [26] by Sun et al.) where the authors formally introduced a generalized inverse called the Moore-Penrose inverse of an even-order tensor via the Einstein product. The authors [26] then used the Moore-Penrose inverse to find the minimum-norm least-squares solution of some multilinear systems. Behera and Mishra [1] continued the same study and proposed different types of generalized inverses in the next year. They initiated the study of the reverse-order law for different generalized inverses of tensors. In 2017, Jin et al. [12] again introduced the Moore-Penrose inverse of a tensor using another tensor product called $t$-product and applied it to derive the higher-order Gauss-Markov theorem. In the same year, Ji and Wei [11] extended the Moore-Penrose inverse of an even-order tensor to the weighted Moore-Penrose inverse of an even-order tensor and established the relation between the minimum-norm least-squares solution of a multilinear system and the weighted Moore-Penrose inverse. In 2018, the same authors introduced another generalized inverse of an even-order square tensor called the Drazin inverse [10]. They used this inverse to find the Drazin inverse solution of the singular linear tensor equation. In the same year, Sun et al. [27] introduced the group inverse of an even-order square tensor using a general product of tensors. Very recently, Huang et al. [9] proposed a tensor-based extreme learning machine. They used the Moore-Penrose inverse to obtain the tensor regression result. In 2018, Panigrahy and Mishra [22] and Stanimirovic et al. [25] simultaneously improved the definition of the Moore-Penrose inverse of an even-order tensor to a tensor of any order via the same product. In 2018, Liang and Zheng [19] also proposed the same definition which appeared in the year 2019. The definition of the Moore-Penrose inverse of an arbitrary order tensor is recalled below.

Definition 1.1 (Definition 1.1, [22]) Let $\mathcal{X} \in \mathbb{C}^{I_{1} \times \cdots \times I_{N} \times J_{1} \times \cdots \times J_{M}}$. The tensor $\mathcal{Y} \in$ $\mathbb{C}^{J_{1} \times \cdots \times J_{M} \times I_{1} \times \cdots \times I_{N}}$ satisfying the following four tensor equations:

$$
\begin{aligned}
\mathcal{X} *_{M} \mathcal{Y} *_{N} \mathcal{X} & =\mathcal{X} \\
\mathcal{Y} *_{N} \mathcal{X} *_{M} \mathcal{Y} & =\mathcal{Y} ; \\
\left(\mathcal{X} *_{M} \mathcal{Y}\right)^{H} & =\mathcal{X} *_{M} \mathcal{Y} ; \\
\left(\mathcal{Y} *_{N} \mathcal{X}\right)^{H} & =\mathcal{Y} *_{N} \mathcal{X}
\end{aligned}
$$

is defined as the Moore-Penrose inverse of $\mathcal{X}$, and is denoted by $\mathcal{X}^{\dagger}$. 
In the above definition, $*_{M}$ denotes the Einstein product [6] of tensors, and is defined by

$$
\left(\mathcal{A} *_{M} \mathcal{B}\right)_{i_{1} \cdots i_{N} k_{1} \cdots k_{L}}=\sum_{j_{1} \cdots j_{M}} a_{i_{1} \cdots i_{N} j_{1} \cdots j_{M}} b_{j_{1} \cdots j_{M} k_{1} \cdots k_{L}}
$$

for tensors $\mathcal{A} \in \mathbb{C}^{I_{1} \times \cdots \times I_{N} \times J_{1} \times \cdots \times J_{M}}$ and $\mathcal{B} \in \mathbb{C}^{J_{1} \times \cdots \times J_{M} \times K_{1} \times \cdots \times K_{L}}$. In the case of an evenorder invertible tensor, the Definition 1.1 coincides with the notion of the inverse which was first introduced by Brazell et al. [4]. They also showed that such an inverse can be computed using the singular value decomposition of the same tensor (see the celebrated result in Lemma 3.1, [4]). A generalized inverse of a tensor, as its name indicates, is a generalization of the notion of the tensor inverse. It is a tensor that exists for a larger class of tensors than the ordinary inverse does; has some properties of the tensor inverse, and for a given even-order square invertible tensor it reduces to the ordinary inverse (see [29] and [31] for theory of generalized inverses of matrices). The idea of introducing generalized inverses of tensors originates from the necessity of finding a solution of a given system of linear equations (see $[1,10,11,26])$.

This paper is in connection with an open problem stated in the last section of [1] for even-order tensors, and is a continuation of the very recent works done in [21] and [22]. The same open problem is restated next in the setting of tensors of any order.

Problem 1. When does $\left(\mathcal{A} *_{N} \mathcal{B}\right)^{\dagger}=\mathcal{B}^{\dagger} *_{N} \mathcal{A}^{\dagger}$ for any two tensors $\mathcal{A} \in \mathbb{C}^{I_{1} \times \cdots \times I_{M} \times J_{1} \times \cdots \times J_{N}}$ and $\mathcal{B} \in \mathbb{C}^{J_{1} \times \cdots \times J_{N} \times K_{1} \times \cdots \times K_{L}}$ ?

Note that Panigrahy et al. [21] attempted first the above Problem 1, very recently, and provided various necessary and sufficient conditions for the same problem, but for evenorder tensors only. Nevertheless, we aim to

1. establish some new necessary and sufficient conditions for the above-stated problem which is well-known as the reverse-order law for the Moore-Penrose inverse of arbitrary order tensors via the Einstein product,

2. compute the Moore-Penrose inverse of the sum of two arbitrary order tensors,

3. find the additive perturbation bounds of the Moore-Penrose inverse under the Frobenius norm, and to

4. introduce the concept of sub-proper splittings for tensors without using the notion of the range and null space of a tensor which may help to find an iterative solution of a tensor multilinear system.

In this context, the paper is organized as follows. Section 2 collects various useful definitions and results. The next section which contains all our main results, partially fulfills the above-stated objectives and provides some open problems for future research interest. It has two subsections. The first subsection deals with the reverse-order law tensors of any order. It also discusses a few properties of different generalized inverses of a tensor, and then illustrates a new expression of the Moore-Penrose inverse of the Einstein product of two tensors in terms of the Einstein product of the Moore-Penrose inverse of a tensor and a generalized inverse of a tensor. The next subsection presents a result which is useful to obtain the additive Moore-Penrose inverse of tensors as an application of the reverse-order law and leaves an open problem. It then attempts the problem of fining the additive perturbation bounds of the Moore-Penrose inverse. Finally, it provides an answer to the last objective of this paper as mentioned in the previous paragraph before moving to the concluding section. 


\section{Prerequisites}

Here, we collect all those remaining definitions and earlier results which will be used to prove the main results in the next section. We begin with the definition of an identity tensor. A tensor $\mathcal{I} \in \mathbb{C}^{I_{1} \times \cdots \times I_{N} \times I_{1} \times \cdots \times I_{N}}$ with entries $(\mathcal{I})_{i_{1} \cdots i_{N} j_{1} \cdots j_{N}}=\prod_{k=1}^{N} \delta_{i_{k} j_{k}}$ is called an identity tensor if $\delta_{i_{k} j_{k}}=\left\{\begin{array}{l}1, \text { if } i_{k}=j_{k} \\ 0, \text { otherwise }\end{array}\right.$. The conjugate transpose of a tensor $\mathcal{A} \in \mathbb{C}^{I_{1} \times \cdots \times I_{M} \times J_{1} \times \cdots \times J_{N}}$ is denoted by $\mathcal{A}^{H}$, and is defined as $\left(\mathcal{A}^{H}\right)_{j_{1} \ldots j_{N} i_{1} \ldots i_{M}}=\bar{a}_{i_{1} \ldots i_{M} j_{1} \ldots j_{N}}$, where the overline stands for the conjugate of $a_{i_{1} \ldots i_{M} j_{1} \ldots j_{N}}$. If the tensor $\mathcal{A}$ is real, then its transpose is denoted by $\mathcal{A}^{T}$, and is defined as $\left(\mathcal{A}^{T}\right)_{j_{1} \ldots j_{N} i_{1} \ldots i_{M}}=a_{i_{1} \ldots i_{M} j_{1} \ldots j_{N}}$. We next present the definition of a unitary and an idempotent tensor. A tensor $\mathcal{A} \in \mathbb{C}^{I_{1} \times \cdots \times I_{N} \times I_{1} \times \cdots \times I_{N}}$ is unitary if $\mathcal{A} *_{N} \mathcal{A}^{H}=\mathcal{A}^{H} *_{N} \mathcal{A}=\mathcal{I}$, and idempotent if $\mathcal{A} *_{N} \mathcal{A}=\mathcal{A}$. If $\mathcal{A}=\mathcal{A}^{H}$ for a tensor $\mathcal{A} \in \mathbb{C}^{I_{1} \times \cdots \times I_{N} \times I_{1} \times \cdots \times I_{N}}$, then $\mathcal{A}$ is Hermitian. If $\mathcal{A}=-\mathcal{A}^{H}$, then it is skew-Hermitian. Liang and Zheng [19] defined a bijective map $\phi$ from the tensor space $\mathbb{C}^{I_{1} \times \cdots \times I_{M} \times J_{1} \times \cdots \times J_{N}}$ into the matrix space $\mathbb{C}^{\left(I_{1} \cdot I_{2} \cdots I_{M}\right) \times\left(J_{1} \cdot J_{2} \cdots J_{N}\right)}$ by

$$
\mathcal{A}=\left(\mathcal{A}_{i_{1} \ldots i_{M} j_{1} \ldots j_{N}}\right) \stackrel{\phi}{\rightarrow} A=\left(A_{i \operatorname{vec}(\mathbf{i}, \mathbb{I}), \operatorname{ivec}(\mathbf{j}, \mathbb{I})}\right),
$$

where

$$
\begin{aligned}
& \operatorname{ivec}(\mathbf{i}, \mathbb{I})=i_{1}+\sum_{r=2}^{M}\left(i_{r}-1\right) \prod_{u=1}^{r-1} I_{u}, \mathbf{i}:=\left\{i_{1}, \cdots, i_{M}\right\}, \mathbb{I}:=\left\{I_{1}, \cdots, I_{M}\right\}, \\
& \operatorname{ivec}(\mathbf{j}, \mathbb{J})=j_{1}+\sum_{s=2}^{N}\left(j_{s}-1\right) \prod_{v=1}^{s-1} J_{v}, \mathbf{j}:=\left\{j_{1}, \cdots, j_{N}\right\}, \mathbb{J}:=\left\{J_{1}, \cdots, J_{N}\right\} .
\end{aligned}
$$

Here, $\mathbb{I}:=\left\{I_{1}, \ldots, I_{M}\right\}$ and $\mathbb{J}:=\left\{J_{1}, \ldots, J_{N}\right\}$ are respectively referred to as the row mode and the column mode of $\mathcal{A}$. Next, we recall the definition of unfolding rank of a tensor.

Definition 2.1 (Definition 3.1, [19]) For a tensor $\mathcal{A} \in \mathbb{C}^{I_{1} \times \cdots \times I_{M} \times J_{1} \times \cdots \times J_{N}}$, and the map $\phi$ as defined in (5), the unfolding rank of the tensor $\mathcal{A}$ is denoted by $\operatorname{rank}_{U}(\mathcal{A})$ and defined as $\operatorname{rank}_{U}(\mathcal{A})=\operatorname{rank}(\phi(\mathcal{A}))$. Particularly, if $\operatorname{rank}_{U}(\mathcal{A})=m\left(\operatorname{rank}_{U}(\mathcal{A})=n\right)$, we say that $\mathcal{A}$ is full row (column) rank, where $m=I_{1} \cdot I_{2} \cdots I_{m}$ and $n=J_{1} \cdot J_{2} \cdots J_{N}$.

The following results are useful to prove our main results. The first one is about the singular value decomposition (SVD) of a tensor proved in Theorem 3.17, [4] for a real tensor. The authors of [26] stated the same result for complex even order tensors. We next present the SVD for arbitrary order tensors.

Lemma 2.2 (Theorem 3.2., [19]) A tensor $\mathcal{A} \in \mathbb{C}^{I_{1} \times \cdots \times I_{M} \times J_{1} \times \cdots \times J_{N}}$ with $\operatorname{rank}_{U}(\mathcal{A})=r$ can be decomposed as

$$
\mathcal{A}=\mathcal{U} *_{M} \mathcal{B} *_{N} \mathcal{V}^{H}
$$

where $\mathcal{U} \in \mathbb{C}^{I_{1} \times \cdots \times I_{M} \times I_{1} \times \cdots \times I_{M}}$ and $\mathcal{V} \in \mathbb{C}^{J_{1} \times \cdots \times J_{N} \times J_{1} \times \cdots \times J_{N}}$ are unitary tensors, and $\mathcal{B} \in \mathbb{R}^{I_{1} \times \cdots \times I_{M} \times J_{1} \times \cdots \times J_{N}}$ is a diagonal tensor defined by

$$
\mathcal{B}_{i_{1} \cdots i_{M} j_{1} \cdots j_{N}}= \begin{cases}\sigma_{i v e c(\mathbf{i}, \mathbb{I})}>0, & \text { ifivec }(\mathbf{i}, \mathbb{I})=\operatorname{ivec}(\mathbf{j}, \mathbb{J}) \in\{1,2, \cdots, r\}, \\ 0, & \text { otherwise, }\end{cases}
$$

where $\sigma_{1} \geq \sigma_{2} \geq \cdots \geq \sigma_{r}>0$ are singular values of the matrix $\phi(\mathcal{A})$. 
The proof of Theorem 3.4, [19] contains the fact that $\mathcal{A}^{\dagger}=\mathcal{V} *_{N} \mathcal{B}^{\dagger} *_{M} \mathcal{U}^{H}$.

Lemma 2.3 (Lemma 2.2, [22]) Let $\mathcal{P} \in \mathbb{C}^{I_{1} \times \cdots \times I_{N} \times I_{1} \times \cdots \times I_{N}}$ be Hermitian. Then

(i) If $\mathcal{P} *_{N} \mathcal{Q}=\mathcal{Q}$ for $\mathcal{Q} \in \mathbb{C}^{I_{1} \times \cdots \times I_{N} \times J_{1} \times \cdots \times J_{M}}$, then $\mathcal{Q}^{\dagger} *_{N} \mathcal{P}=\mathcal{Q}^{\dagger}$.

(ii) If $\mathcal{Q} *_{N} \mathcal{P}=\mathcal{Q}$ for $\mathcal{Q} \in \mathbb{C}^{J_{1} \times \cdots \times J_{M} \times I_{1} \times \cdots \times I_{N}}$, then $\mathcal{P} *_{N} \mathcal{Q}^{\dagger}=\mathcal{Q}^{\dagger}$.

Next, we restate a few results for tensors (arbitrary order) which are proved by Panigrahy et al. [21] very recently for even-order tensors. The very first result stated below extends Theorem 3.25, [21] to any two tensors, and provides necessary and sufficient conditions for the reverse-order law which is mentioned in Problem 1 in the introduction section.

Theorem 2.4 Let $\mathcal{A} \in \mathbb{C}^{I_{1} \times \cdots \times I_{M} \times J_{1} \times \cdots \times J_{N}}$ and $\mathcal{B} \in \mathbb{C}^{J_{1} \times \cdots \times J_{N} \times K_{1} \times \cdots \times K_{L}}$. Then $\left(\mathcal{A} *_{N} \mathcal{B}\right)^{\dagger}=\mathcal{B}^{\dagger} *_{N} \mathcal{A}^{\dagger}$ if and only if

$$
\mathcal{A}^{\dagger} *_{M} \mathcal{A} *_{N} \mathcal{B} *_{L} \mathcal{B}^{H} *_{N} \mathcal{A}^{H}=\mathcal{B} *_{L} \mathcal{B}^{H} *_{N} \mathcal{A}^{H}
$$

and

$$
\mathcal{B} *_{L} \mathcal{B}^{\dagger} *_{N} \mathcal{A}^{H} *_{M} \mathcal{A} *_{N} \mathcal{B}=\mathcal{A}^{H} *_{M} \mathcal{A} *_{N} \mathcal{B}
$$

The next two lemmas are again the modified version of Lemma 2.5 and Lemma 2.6, [21], and are frequently used to derive some new reverse-order laws. The first one provides a necessary and sufficient condition for the commutative property of $\mathcal{A}^{\dagger} *_{M} \mathcal{A}$ and $\mathcal{B} *_{L} \mathcal{B}^{H}$.

Lemma 2.5 Let $\mathcal{A} \in \mathbb{C}^{I_{1} \times \cdots \times I_{M} \times J_{1} \times \cdots \times J_{N}}$ and $\mathcal{B} \in \mathbb{C}^{J_{1} \times \cdots \times J_{N} \times K_{1} \times \cdots \times K_{L}}$. Then,

$$
\mathcal{A}^{\dagger} *_{M} \mathcal{A} *_{N} \mathcal{B} *_{L} \mathcal{B}^{H} *_{N} \mathcal{A}^{H}=\mathcal{B} *_{L} \mathcal{B}^{H} *_{N} \mathcal{A}^{H}
$$

if and only if $\mathcal{A}^{\dagger} *_{M} \mathcal{A}$ commutes with $\mathcal{B} *_{L} \mathcal{B}^{H}$.

Similarly, the next one presents a sufficient condition for the commutative property of $\mathcal{A}^{H} *_{M} \mathcal{A}$ and $\mathcal{B} *_{L} \mathcal{B}^{\dagger}$.

Lemma 2.6 Let $\mathcal{A} \in \mathbb{C}^{I_{1} \times \cdots \times I_{M} \times J_{1} \times \cdots \times J_{N}}$ and $\mathcal{B} \in \mathbb{C}^{J_{1} \times \cdots \times J_{N} \times K_{1} \times \cdots \times K_{L}}$. Then,

$$
\mathcal{B} *_{L} \mathcal{B}^{\dagger} *_{N} \mathcal{A}^{H} *_{M} \mathcal{A} *_{N} \mathcal{B}=\mathcal{A}^{H} *_{M} \mathcal{A} *_{N} \mathcal{B}
$$

if and only if $\mathcal{A}^{H} *_{M} \mathcal{A}$ commutes with $\mathcal{B} *_{L} \mathcal{B}^{\dagger}$.

Theorem 2.4 together with Lemma 2.5 and Lemma 2.6 yields the following outcome.

Theorem 2.7 Let $\mathcal{A} \in \mathbb{C}^{I_{1} \times \cdots \times I_{M} \times J_{1} \times \cdots \times J_{N}}$ and $\mathcal{B} \in \mathbb{C}^{J_{1} \times \cdots \times J_{N} \times K_{1} \times \cdots \times K_{L}}$. Then $\left(\mathcal{A} *_{N} \mathcal{B}\right)^{\dagger}=\mathcal{B}^{\dagger} *_{N} \mathcal{A}^{\dagger}$ if and only if $\mathcal{A}^{\dagger} *_{M} \mathcal{A}$ commutes with $\mathcal{B} *_{L} \mathcal{B}^{H}$ and $\mathcal{A}^{H} *_{M} \mathcal{A}$ commutes with $\mathcal{B} *_{L} \mathcal{B}^{\dagger}$.

Theorem 3.30, [21] is reproduced here for any two tensors.

Theorem 2.8 Let $\mathcal{A} \in \mathbb{C}^{I_{1} \times \cdots \times I_{M} \times J_{1} \times \cdots \times J_{N}}$ and $\mathcal{B} \in \mathbb{C}^{J_{1} \times \cdots \times J_{N} \times K_{1} \times \cdots \times K_{L}}$. If $\left(\mathcal{A} *_{N} \mathcal{B}\right)^{\dagger}=$ $\mathcal{B}^{\dagger} *_{N} \mathcal{A}^{\dagger}$, then $\mathcal{A}^{\dagger} *_{M} \mathcal{A}$ and $\mathcal{B} *_{L} \mathcal{B}^{\dagger}$ commute.

In general, the converse of the above result doesn't hold (see Example 3.31, [21]). 


\begin{tabular}{cc|cc|cc}
\hline \multicolumn{2}{c}{$\left(\mathcal{A} *_{1} \mathcal{B}\right)^{\dagger}(:,:, 1)$} & \multicolumn{2}{c}{$\left(\mathcal{A} *_{1} \mathcal{B}\right)^{\dagger}(:,:, 2)$} & \multicolumn{2}{c}{$\left(\mathcal{A} *_{1} \mathcal{B}\right)^{\dagger}(:,:, 3)$} \\
\hline $6 / 17$ & 0 & $-2 / 17$ & $1 / 17$ & $3 / 17$ & $1 / 17$ \\
$-4 / 17$ & 0 & $7 / 17$ & $5 / 17$ & $-2 / 17$ & $5 / 17$ \\
\hline
\end{tabular}

So, we get $\mathcal{B}^{\dagger} *_{1} \mathcal{A}^{\dagger} \in \mathbb{C}^{2 \times 2 \times 3}$, where

\begin{tabular}{cc|cc|cc}
\hline \multicolumn{2}{c}{$\mathcal{B}^{\dagger} *_{1} \mathcal{A}^{\dagger}(:,:, 1)$} & \multicolumn{2}{c}{$\mathcal{B}^{\dagger} *_{1} \mathcal{A}^{\dagger}(:,:, 2)$} & \multicolumn{1}{c}{$\mathcal{B}^{\dagger} *_{1} \mathcal{A}^{\dagger}(:,:, 3)$} \\
\hline $3 / 8$ & 0 & $-1 / 8$ & 0 & $1 / 8$ & $1 / 8$ \\
$-1 / 4$ & 0 & $1 / 4$ & $1 / 2$ & $-1 / 4$ & $1 / 4$ \\
\hline
\end{tabular}

We thus have

$$
\mathcal{B} *_{1} \mathcal{B}^{\dagger} *_{1} \mathcal{A}^{\dagger} *_{2} \mathcal{A}(:,:)=\left(\begin{array}{cccc}
1 / 2 & 0 & 1 / 2 & 0 \\
0 & 1 / 2 & 0 & 1 / 2 \\
1 / 2 & 0 & 1 / 2 & 0 \\
0 & 1 / 2 & 0 & 1 / 2
\end{array}\right), \quad \mathcal{A}^{\dagger} *_{2} \mathcal{A} *_{1} \mathcal{B} *_{1} \mathcal{B}^{\dagger}(:,:)=\left(\begin{array}{cccc}
1 / 2 & 0 & 1 / 2 & 0 \\
0 & 1 / 2 & 0 & 1 / 2 \\
1 / 2 & 0 & 1 / 2 & 0 \\
0 & 1 / 2 & 0 & 1 / 2
\end{array}\right),
$$

and

$$
\left(\mathcal{A}^{\dagger} *_{2} \mathcal{A} *_{1} \mathcal{B} *_{1} \mathcal{B}^{\dagger}\right)^{\dagger}(:,:)=\left(\begin{array}{cccc}
1 / 2 & 0 & 1 / 2 & 0 \\
0 & 1 / 2 & 0 & 1 / 2 \\
1 / 2 & 0 & 1 / 2 & 0 \\
0 & 1 / 2 & 0 & 1 / 2
\end{array}\right) .
$$

Whence, $\left(\mathcal{A}^{\dagger} *_{2} \mathcal{A} *_{1} \mathcal{B} *_{1} \mathcal{B}^{\dagger}\right)^{\dagger}=\mathcal{B} *_{1} \mathcal{B}^{\dagger} *_{1} \mathcal{A}^{\dagger} *_{2} \mathcal{A}$, but $\left(\mathcal{A} *_{1} \mathcal{B}\right)^{\dagger} \neq \mathcal{B}^{\dagger} *_{1} \mathcal{A}^{\dagger}$.

The converse of Lemma 3.1 is true under the assumption of the fact that $\mathcal{A} *_{N} \mathcal{B} *_{L} \mathcal{B}^{\dagger} *_{N} \mathcal{A}^{\dagger}$ and $\mathcal{B}^{\dagger} *_{N} \mathcal{A}^{\dagger} *_{M} \mathcal{A} *_{N} \mathcal{B}$ are Hermitian. The stated fact is proved below.

Lemma 3.3 Let $\mathcal{A} \in \mathbb{C}^{I_{1} \times \cdots \times I_{M} \times J_{1} \times \cdots \times J_{N}}$ and $\mathcal{B} \in \mathbb{C}^{J_{1} \times \cdots \times J_{N} \times K_{1} \times \cdots \times K_{L}}$. Also, let $\mathcal{P}=$ $\mathcal{A}^{\dagger} *_{M} \mathcal{A}$ and $\mathcal{Q}=\mathcal{B} *_{L} \mathcal{B}^{\dagger}$. If $\mathcal{P} *_{N} \mathcal{Q}=\mathcal{Q} *_{N} \mathcal{P},\left(\mathcal{A} *_{N} \mathcal{B} *_{L} \mathcal{B}^{\dagger} *_{N} \mathcal{A}^{\dagger}\right)^{H}=\mathcal{A} *_{N} \mathcal{B} *_{L} \mathcal{B}^{\dagger} *_{N} \mathcal{A}^{\dagger}$ and $\left(\mathcal{B}^{\dagger} *_{N} \mathcal{A}^{\dagger} *_{M} \mathcal{A} *_{N} \mathcal{B}\right)^{H}=\mathcal{B}^{\dagger} *_{N} \mathcal{A}^{\dagger} *_{M} \mathcal{A} *_{N} \mathcal{B}$, then $\left(\mathcal{A} *_{N} \mathcal{B}\right)^{\dagger}=\mathcal{B}^{\dagger} *_{N} \mathcal{A}^{\dagger}$.

Proof Since $\mathcal{P} *_{N} \mathcal{Q}=\mathcal{Q} *{ }_{N} \mathcal{P}$, so by Eq. (1) we get

$$
\mathcal{A}^{\dagger} *_{M} \mathcal{A} *_{N} \mathcal{B} *_{L} \mathcal{B}^{\dagger} *_{N} \mathcal{A}^{\dagger} *_{M} \mathcal{A} *_{N} \mathcal{B} *_{L} \mathcal{B}^{\dagger}=\mathcal{A}^{\dagger} *_{M} \mathcal{A} *_{N} \mathcal{B} *_{L} \mathcal{B}^{\dagger}
$$

and by Eq. (2) we get

$$
\mathcal{B} *_{L} \mathcal{B}^{\dagger} *_{N} \mathcal{A}^{\dagger} *_{M} \mathcal{A} *_{N} \mathcal{B} *_{L} \mathcal{B}^{\dagger} *_{N} \mathcal{A}^{\dagger} *_{M} \mathcal{A}=\mathcal{B} *_{L} \mathcal{B}^{\dagger} *_{N} \mathcal{A}^{\dagger} *_{M} \mathcal{A}
$$

Let $\mathcal{X}=\mathcal{A} *_{N} \mathcal{B}$ and $\mathcal{Y}=\mathcal{B}^{\dagger} *_{N} \mathcal{A}^{\dagger}$. Pre-multiplying $\mathcal{A}$ and post-multiplying $\mathcal{B}$ to Eq. (8) yields $\mathcal{X} *_{L} \mathcal{Y} *_{M} \mathcal{X}=\mathcal{X}$, and on pre-multiplication of $\mathcal{B}^{\dagger}$ and post-multiplication of $\mathcal{A}^{\dagger}$ with Eq. (9) results $\mathcal{Y} *_{M} \mathcal{X} *_{L} \mathcal{Y}=\mathcal{Y}$. The Hermitian property of $\mathcal{X} *_{L} \mathcal{Y}$ and $\mathcal{Y} *_{M} \mathcal{X}$ are confirmed by the second and third assumptions. The claim is thus attained by Definition 1.1.

Theorem 2.8 and Lemma 3.3 can now be together stated as following.

Theorem 3.4 Let $\mathcal{A} \in \mathbb{C}^{I_{1} \times \cdots \times I_{M} \times J_{1} \times \cdots \times J_{N}}$ and $\mathcal{B} \in \mathbb{C}^{J_{1} \times \cdots \times J_{N} \times K_{1} \times \cdots \times K_{L}}$. Then $\left(\mathcal{A} *_{N} \mathcal{B}\right)^{\dagger}=\mathcal{B}^{\dagger} *_{N} \mathcal{A}^{\dagger}$ if and only if

(i) $\mathcal{A}^{\dagger} *_{M} \mathcal{A} *_{N} \mathcal{B} *_{L} \mathcal{B}^{\dagger}=\mathcal{B} *_{L} \mathcal{B}^{\dagger} *_{N} \mathcal{A}^{\dagger} *_{M} \mathcal{A}$,

(ii) $\left(\mathcal{A} *_{N} \mathcal{B} *_{L} \mathcal{B}^{\dagger} *_{N} \mathcal{A}^{\dagger}\right)^{H}=\mathcal{A} *_{N} \mathcal{B} *_{L} \mathcal{B}^{\dagger} *_{N} \mathcal{A}^{\dagger}$,

(iii) $\left(\mathcal{B}^{\dagger} *_{N} \mathcal{A}^{\dagger} *_{M} \mathcal{A} *_{N} \mathcal{B}\right)^{H}=\mathcal{B}^{\dagger} *_{N} \mathcal{A}^{\dagger} *_{M} \mathcal{A} *_{N} \mathcal{B}$.

Now, we move to the proof an interesting result which is helpful to improve the reverseorder law. 
Lemma 3.5 Let $\mathcal{A} \in \mathbb{C}^{I_{1} \times \cdots \times I_{M} \times J_{1} \times \cdots \times J_{N}}$ and $\mathcal{B} \in \mathbb{C}^{J_{1} \times \cdots \times J_{N} \times K_{1} \times \cdots \times K_{L}}$. If $\mathcal{A}^{\dagger} *_{M} \mathcal{A} *_{N} \mathcal{B}$ $*_{L} \mathcal{B}^{H} *_{N} \mathcal{A}^{H}=\mathcal{B} *_{L} \mathcal{B}^{H} *_{N} \mathcal{A}^{H}$, then $\mathcal{B}^{\dagger} *_{N} \mathcal{A}^{\dagger} \in\left(\mathcal{A} *_{N} \mathcal{B}\right)\{1,2,4\}$.

Proof Pre-multiplying and post-multiplying $\mathcal{A}^{\dagger} *_{M} \mathcal{A} *_{N} \mathcal{B} *_{L} \mathcal{B}^{H} *_{N} \mathcal{A}^{H}=\mathcal{B} *_{L} \mathcal{B}^{H} *_{N} \mathcal{A}^{H}$ by $\mathcal{B}^{\dagger}$ and $\left(\left(\mathcal{A} *_{N} \mathcal{B}\right)^{H}\right)^{\dagger}$, respectively, we get

$$
\begin{aligned}
\mathcal{B}^{\dagger} *_{N} \mathcal{A}^{\dagger} *_{M} \mathcal{A} *_{N} \mathcal{B} *_{L} \mathcal{B}^{H} *_{N} \mathcal{A}^{H} *_{M}\left(\left(\mathcal{A} *_{N} \mathcal{B}\right)^{H}\right)^{\dagger} & =\mathcal{B}^{\dagger} *_{N} \mathcal{B} *_{L} \mathcal{B}^{H} *_{N} \mathcal{A}^{H} *_{M}\left(\left(\mathcal{A} *_{N} \mathcal{B}\right)^{H}\right)^{\dagger} \\
& =\left(\mathcal{A} *_{N} \mathcal{B}\right)^{\dagger} *_{M} \mathcal{A} *_{N} \mathcal{B} .
\end{aligned}
$$

But

$$
\begin{aligned}
\mathcal{B}^{\dagger} *_{N} \mathcal{A}^{\dagger} *_{M} \mathcal{A} *_{N} \mathcal{B} *_{L} \mathcal{B}^{H} *_{N} \mathcal{A}^{H} *_{M}\left(\left(\mathcal{A} *_{N} \mathcal{B}\right)^{H}\right)^{\dagger} & =\mathcal{B}^{\dagger} *_{N} \mathcal{A}^{\dagger} *_{M} \mathcal{A} *_{N} \mathcal{B} *_{L}\left(\mathcal{A} *_{N} \mathcal{B}\right)^{H} *_{M}\left(\left(\mathcal{A} *_{N} \mathcal{B}\right)^{\dagger}\right)^{H} \\
& =\mathcal{B}^{\dagger} *_{N} \mathcal{A}^{\dagger} *_{M} \mathcal{A} *_{N} \mathcal{B} .
\end{aligned}
$$

Thus, we have

$$
\mathcal{B}^{\dagger} *_{N} \mathcal{A}^{\dagger} *_{M} \mathcal{A} *_{N} \mathcal{B}=\left(\mathcal{A} *_{N} \mathcal{B}\right)^{\dagger} *_{M} \mathcal{A} *_{N} \mathcal{B} .
$$

Let $\mathcal{X}=\mathcal{A} *_{N} \mathcal{B}$ and $\mathcal{Y}=\mathcal{B}^{\dagger} *_{N} \mathcal{A}^{\dagger}$. Then pre-multiplying $\mathcal{A} *_{N} \mathcal{B}$ to Eq. (10), we get $\mathcal{X} *_{L} \mathcal{Y} *_{M} \mathcal{X}=\mathcal{X}$. Using Lemma 2.5, we have

$$
\mathcal{B} *_{L} \mathcal{B}^{H} *_{N} \mathcal{A}^{\dagger} *_{M} \mathcal{A} *_{N} \mathcal{B} *_{L} \mathcal{B}^{\dagger} *_{N} \mathcal{A}^{H}=\mathcal{B} *_{L} \mathcal{B}^{H} *_{N} \mathcal{A}^{H} .
$$

Pre-multiplying $\mathcal{B}^{\dagger}$ to the above equation, we obtain

$$
\mathcal{B}^{H} *_{N} \mathcal{A}^{\dagger} *_{M} \mathcal{A} *_{N} \mathcal{B} *_{L} \mathcal{B}^{\dagger} *_{N} \mathcal{A}^{H}=\mathcal{B}^{H} *_{N} \mathcal{A}^{H}
$$

which reduces to

$$
\mathcal{B} *_{L} \mathcal{B}^{\dagger} *_{N} \mathcal{A}^{\dagger} *_{M} \mathcal{A} *_{N} \mathcal{B} *_{L} \mathcal{B}^{\dagger} *_{N} \mathcal{A}^{\dagger} *_{M} \mathcal{A}=\mathcal{B} *_{L} \mathcal{B}^{\dagger} *_{N} \mathcal{A}^{\dagger} *_{M} \mathcal{A}
$$

by pre-multiplying $\mathcal{B}^{\dagger H}$ and post-multiplying $\mathcal{A}^{\dagger H}$ again, simultaneously. The above equation gives $\mathcal{Y} *_{M} \mathcal{X} *_{L} \mathcal{Y}=\mathcal{Y}$ by pre-multiplying $\mathcal{B}^{\dagger}$ and post-multiplying $\mathcal{A}^{\dagger}$. The Hermitian property of $\mathcal{Y} *_{M} \mathcal{X}$ can be proved by using Eq. (10). Thus, $\mathcal{Y} \in \mathcal{X}\left\{1,2\right.$, 4\}, i.e., $\mathcal{B}^{\dagger} *_{N} \mathcal{A}^{\dagger} \in$ $\left(\mathcal{A} *_{N} \mathcal{B}\right)\{1,2,4\}$ by Definition 1.1 .

It can also be proved that if $\mathcal{B} *_{L} \mathcal{B}^{\dagger} *_{N} \mathcal{A}^{H} *_{M} \mathcal{A} *_{N} \mathcal{B}=\mathcal{A}^{H} *_{M} \mathcal{A} *_{N} \mathcal{B}$, then $\mathcal{B}^{\dagger} *_{N} \mathcal{A}^{\dagger} \in$ $\left(\mathcal{A} *_{N} \mathcal{B}\right)\{1,2,3\}$. Based on the above fact, Theorem 2.4 can now be improved, and the improved version is presented next.

Theorem 3.6 Let $\mathcal{A} \in \mathbb{C}^{I_{1} \times \cdots \times I_{M} \times J_{1} \times \cdots \times J_{N}}$ and $\mathcal{B} \in \mathbb{C}^{J_{1} \times \cdots \times J_{N} \times K_{1} \times \cdots \times K_{L}}$. Then $\left(\mathcal{A} *{ }_{N} \mathcal{B}\right)^{\dagger}=\mathcal{B}^{\dagger} *_{N} \mathcal{A}^{\dagger}$ if and only if

(i) $\mathcal{A}^{\dagger} *_{M} \mathcal{A} *_{N} \mathcal{B} *_{L} \mathcal{B}^{H} *_{N} \mathcal{A}^{H}=\mathcal{B} *_{L} \mathcal{B}^{H} *_{N} \mathcal{A}^{H}$,

(ii) $\mathcal{A} *_{N} \mathcal{B} *_{L} \mathcal{B}^{\dagger} *_{N} \mathcal{A}^{\dagger}$ is Hermitian.

Proof Let $\left(\mathcal{A} *_{N} \mathcal{B}\right)^{\dagger}=\mathcal{B}^{\dagger} *_{N} \mathcal{A}^{\dagger}$. So $\mathcal{A} *_{N} \mathcal{B} *_{L} \mathcal{B}^{\dagger} *_{N} \mathcal{A}^{\dagger}=\mathcal{A} *_{N} \mathcal{B} *_{L}\left(\mathcal{A} *_{N} \mathcal{B}\right)^{\dagger}$ is Hermitian by the definition of the Moore-Penrose inverse of a tensor. By Theorem 2.7, we have $(i)$.

Conversely, $(i)$ implies $\mathcal{B}^{\dagger} *_{N} \mathcal{A}^{\dagger} \in\left(\mathcal{A} *_{N} \mathcal{B}\right)\{1,2,4\}$ by Lemma 3.5 and in addition with condition $(i i)$ the claim is justified.

Now, another improved version of Theorem 2.4 can be stated as $\left(\mathcal{A} *_{N} \mathcal{B}\right)^{\dagger}=\mathcal{B}^{\dagger} *_{N} \mathcal{A}^{\dagger}$ if and only if $\mathcal{B} *_{L} \mathcal{B}^{\dagger} *_{N} \mathcal{A}^{H} *_{M} \mathcal{A} *_{N} \mathcal{B}=\mathcal{A}^{H}{ }{ }_{M} \mathcal{A} *_{N} \mathcal{B}$, and $\mathcal{B}^{\dagger} *_{N} \mathcal{A}^{\dagger} *_{M} \mathcal{A} *_{N} \mathcal{B}$ is Hermitian. A new characterization of the reverse-order law is proved next.

Theorem 3.7 Let $\mathcal{A} \in \mathbb{C}^{I_{1} \times \cdots \times I_{M} \times J_{1} \times \cdots \times J_{N}}$ and $\mathcal{B} \in \mathbb{C}^{J_{1} \times \cdots \times J_{N} \times K_{1} \times \cdots \times K_{L}}$. Then $\left(\mathcal{A} *_{N} \mathcal{B}\right)^{\dagger}=\mathcal{B}^{\dagger} *_{N} \mathcal{A}^{\dagger}$ is equivalent to 
(i) $\left(\mathcal{A}^{\dagger} *_{M} \mathcal{A} *_{N} \mathcal{B}\right)^{\dagger}=\mathcal{B}^{\dagger} *_{N} \mathcal{A}^{\dagger} *_{M} \mathcal{A}$,

(ii) $\left(\mathcal{A} *_{N} \mathcal{B}\right)^{\dagger}=\left(\mathcal{A}^{\dagger} *_{M} \mathcal{A} *_{N} \mathcal{B}\right)^{\dagger} *_{N} \mathcal{A}^{\dagger}$.

Proof Suppose that $\left(\mathcal{A} *_{N} \mathcal{B}\right)^{\dagger}=\mathcal{B}^{\dagger} *_{N} \mathcal{A}^{\dagger}$. By Theorem 2.7, $\mathcal{A}^{\dagger} *_{M} \mathcal{A}$ commutes with $\mathcal{B} *_{L} \mathcal{B}^{H}$ and $\mathcal{A}^{H} *_{M} \mathcal{A}$ commutes with $\mathcal{B} *_{L} \mathcal{B}^{\dagger}$. Let $\mathcal{X}=\mathcal{A}^{\dagger} *_{M} \mathcal{A}$ and $\mathcal{Y}=\mathcal{B}$. Then a simple calculation leads to $\mathcal{X}^{\dagger} *_{N} \mathcal{X} *_{N} \mathcal{Y} *_{L} \mathcal{Y}^{H}=\mathcal{Y} *_{L} \mathcal{Y}^{H} *_{N} \mathcal{X}^{\dagger} *_{N} \mathcal{X}$. By Theorem 2.8, we also have $\mathcal{Y} *_{L} \mathcal{Y}^{\dagger} *_{N} \mathcal{X}^{H} *_{N} \mathcal{X}=\mathcal{X}^{H_{*_{N}}} \mathcal{X} *_{N} \mathcal{Y} *_{L} \mathcal{Y}^{\dagger}$. By Theorem 2.7, we thus have $\left(\mathcal{X} *_{N} \mathcal{Y}\right)^{\dagger}=\mathcal{Y}^{\dagger} *_{N} \mathcal{X}^{\dagger}$, i.e., $\left(\mathcal{A}^{\dagger} *_{M} \mathcal{A} *_{N} \mathcal{B}\right)^{\dagger}=\mathcal{B}^{\dagger} *_{N} \mathcal{A}^{\dagger} *_{M} \mathcal{A}$, which on post-multiplication of $\mathcal{A}^{\dagger}$ yields (ii). The converse part is straightforward.

Similarly, one can prove that $\left(\mathcal{A} *_{N} \mathcal{B}\right)^{\dagger}=\mathcal{B}^{\dagger} *_{N} \mathcal{A}^{\dagger}$ is equivalent to $\left(\mathcal{A} *_{N} \mathcal{B} *_{L} \mathcal{B}^{\dagger}\right)^{\dagger}=$ $\mathcal{B} *_{L} \mathcal{B}^{\dagger} *_{N} \mathcal{A}^{\dagger}$ and $\left(\mathcal{A} *_{N} \mathcal{B}\right)^{\dagger}=\mathcal{B}^{\dagger} *_{N}\left(\mathcal{A} *_{N} \mathcal{B} *_{L} \mathcal{B}^{\dagger}\right)^{\dagger}$. Next result extends Theorem 3.1, [21] for any tensor, and is useful in proving Theorems 3.9, 3.10 and 3.11. The proof follows parallelly from the corresponding proof given in [21].

Lemma 3.8 Let $\mathcal{A} \in \mathbb{C}^{I_{1} \times \cdots \times I_{M} \times J_{1} \times \cdots \times J_{N}}$. Then

(a) $\left(\mathcal{A}^{H} *_{M} \mathcal{A}\right)^{\dagger}=\mathcal{A}^{\dagger} *_{M}\left(\mathcal{A}^{H}\right)^{\dagger}$;

(b) $\left(\mathcal{A} *_{N} \mathcal{A}^{H}\right)^{\dagger}=\left(\mathcal{A}^{H}\right)^{\dagger} *_{N} \mathcal{A}^{\dagger}$.

The above result helps us to obtain two more equivalent conditions of the reverse-order law.

Theorem 3.9 Let $\mathcal{A} \in \mathbb{C}^{I_{1} \times \cdots \times I_{M} \times J_{1} \times \cdots \times J_{N}}$ and $\mathcal{B} \in \mathbb{C}^{J_{1} \times \cdots \times J_{N} \times K_{1} \times \cdots \times K_{L}}$. Then $\left(\mathcal{A} *_{N} \mathcal{B}\right)^{\dagger}=\mathcal{B}^{\dagger} *_{N} \mathcal{A}^{\dagger}$ is equivalent to

(i) $\left(\mathcal{A}^{H} *_{M} \mathcal{A} *_{N} \mathcal{B}\right)^{\dagger}=\mathcal{B}^{\dagger} *_{N}\left(\mathcal{A}^{H} *_{M} \mathcal{A}\right)^{\dagger}$,

(ii) $\left(\mathcal{A} *_{N} \mathcal{B}\right)^{\dagger}=\left(\mathcal{A}^{H} *_{M} \mathcal{A} *_{N} \mathcal{B}\right)^{\dagger} *_{N} \mathcal{A}^{H}$.

Proof Let $\left(\mathcal{A} *_{N} \mathcal{B}\right)^{\dagger}=\mathcal{B}^{\dagger} *_{N} \mathcal{A}^{\dagger}$. So, $\mathcal{A}^{\dagger} *_{M} \mathcal{A}$ commutes with $\mathcal{B} *_{L} \mathcal{B}^{H}$ and $\mathcal{A}^{H} *_{M} \mathcal{A}$ commutes with $\mathcal{B} *_{L} \mathcal{B}^{\dagger}$ due to Theorem 2.7. Let $\mathcal{X}=\mathcal{A}^{H} *_{M} \mathcal{A}$ and $\mathcal{Y}=\mathcal{B}$. Clearly, $\mathcal{X}$ is Hermitian and also observe that $\mathcal{X}^{\dagger} *_{N} \mathcal{X}=\mathcal{A}^{\dagger} *_{M} \mathcal{A}$ due to Lemma 3.8. So, $\mathcal{X}^{\dagger} *_{N} \mathcal{X}$ commutes with $\mathcal{Y} *_{L} \mathcal{Y}^{H}$ and $\mathcal{X}^{H} *_{N} \mathcal{X}$ commutes with $\mathcal{Y} *_{L} \mathcal{Y}^{\dagger}$. Thus, $\left(\mathcal{X} *_{N} \mathcal{Y}\right)^{\dagger}=\mathcal{Y}^{\dagger} *_{N} \mathcal{X}^{\dagger}$, i.e., $\left(\mathcal{A}^{H} *_{M} \mathcal{A} *_{N} \mathcal{B}\right)^{\dagger}=\mathcal{B}^{\dagger} *_{N}\left(\mathcal{A}^{H} *_{M} \mathcal{A}\right)^{\dagger}$. Now, on post-multiplying $\mathcal{A}^{H}$ to $(i)$ we obtain $(i i)$. Converse part is straightforward.

Analogously, another characterization of the reverse-order law is stated next. $\left(\mathcal{A} *_{N} \mathcal{B}\right)^{\dagger}=$ $\mathcal{B}^{\dagger} *_{N} \mathcal{A}^{\dagger}$ is equivalent to $\left(\mathcal{A} *_{N} \mathcal{B} *_{L} \mathcal{B}^{H}\right)^{\dagger}=\left(\mathcal{B} *_{L} \mathcal{B}^{H}\right)^{\dagger} *_{N} \mathcal{A}^{\dagger}$ and $\left(\mathcal{A} *_{N} \mathcal{B}\right)^{\dagger}=\mathcal{B}^{H} *_{N}$ $\left(\mathcal{A} *_{N} \mathcal{B} *_{L} \mathcal{B}^{H}\right)^{\dagger}$. We provide below another set of new necessary and sufficient conditions for the reverse-order law.

Theorem 3.10 Let $\mathcal{A} \in \mathbb{C}^{I_{1} \times \cdots \times I_{M} \times J_{1} \times \cdots \times J_{N}}$ and $\mathcal{B} \in \mathbb{C}^{J_{1} \times \cdots \times J_{N} \times K_{1} \times \cdots \times K_{L}}$. Then $\left(\mathcal{A} *_{N} \mathcal{B}\right)^{\dagger}=\mathcal{B}^{\dagger} *_{N} \mathcal{A}^{\dagger}$ is equivalent to

(i) $\left(\mathcal{A}^{H} *_{M} \mathcal{A} *_{N} \mathcal{B} *_{L} \mathcal{B}^{H}\right)^{\dagger}=\left(\mathcal{B} *_{L} \mathcal{B}^{H}\right)^{\dagger} *_{N}\left(\mathcal{A}^{H} *_{M} \mathcal{A}\right)^{\dagger}$,

(ii) $\left(\mathcal{A} *_{N} \mathcal{B}\right)^{\dagger}=\mathcal{B}^{H} *_{N}\left(\mathcal{A}^{H} *_{M} \mathcal{A} *_{N} \mathcal{B} *_{L} \mathcal{B}^{H}\right)^{\dagger} *_{N} \mathcal{A}^{H}$.

Proof Suppose that $\left(\mathcal{A} *_{N} \mathcal{B}\right)^{\dagger}=\mathcal{B}^{\dagger} *_{N} \mathcal{A}^{\dagger}$, then $\mathcal{A}^{\dagger} *_{M} \mathcal{A}$ commutes with $\mathcal{B} *_{L} \mathcal{B}^{H}$ and $\mathcal{A}^{H} *_{M} \mathcal{A}$ commutes with $\mathcal{B} *_{L} \mathcal{B}^{\dagger}$. Let $\mathcal{X}=\mathcal{A}^{H} *_{M} \mathcal{A}$ and $\mathcal{Y}=\mathcal{B} *_{L} \mathcal{B}^{H}$. In view of the Lemma 3.8, we have $\mathcal{X}^{\dagger} *_{N} \mathcal{X}=\mathcal{A}^{\dagger} *_{M} \mathcal{A}$ and $\mathcal{Y} *_{N} \mathcal{Y}^{\dagger}=\mathcal{B} *_{L} \mathcal{B}^{\dagger}$. So, it can be easily seen that $\mathcal{X}^{\dagger} *_{N} \mathcal{X}$ commutes with $\mathcal{Y} *_{N} \mathcal{Y}^{H}$ and $\mathcal{X}^{H} *_{N} \mathcal{X}$ commutes with $\mathcal{Y}_{*_{N}} \mathcal{Y}^{\dagger}$. Thus, $\left(\mathcal{X} *_{N} \mathcal{Y}\right)^{\dagger}=\mathcal{Y}^{\dagger} *_{N} \mathcal{X}^{\dagger}$, i.e., $\left(\mathcal{A}^{H} *_{M} \mathcal{A} *_{N} \mathcal{B} *_{L} \mathcal{B}^{H}\right)^{\dagger}=\left(\mathcal{B} *_{L} \mathcal{B}^{H}\right)^{\dagger} *_{N}\left(\mathcal{A}^{H} *_{M} \mathcal{A}\right)^{\dagger}$ due to Theorem 2.7. Pre-multiplying $\mathcal{B}^{H}$ and post-multiplying $\mathcal{A}^{H}$ to $(i)$ yields $(i i)$. Converse part is straightforward. 
It can be easily shown that for any tensor $\mathcal{A} \in \mathbb{C}^{I_{1} \times \cdots \times I_{M} \times J_{1} \times \cdots \times J_{N}}$, we have

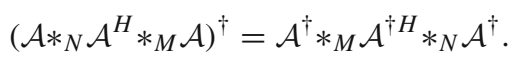

The aforementioned equality aims to achieve the following equality.

$$
\begin{aligned}
& \mathcal{A} *_{N} \mathcal{A}^{H} *_{M} \mathcal{A} *_{N}\left(\mathcal{A} *_{N} \mathcal{A}^{H} *_{M} \mathcal{A}\right)^{\dagger}=\mathcal{A} *_{N} \mathcal{A}^{\dagger}, \\
& \left(\mathcal{A} *_{N} \mathcal{A}^{H} *_{M} \mathcal{A}\right)^{\dagger} *_{M} \mathcal{A} *_{N} \mathcal{A}^{H} *_{M} \mathcal{A}=\mathcal{A}^{\dagger} *_{M} \mathcal{A},
\end{aligned}
$$

for any tensor $\mathcal{A} \in \mathbb{C}^{I_{1} \times \cdots \times I_{M} \times J_{1} \times \cdots \times J_{N}}$. Using Eqs. (11) and (12), we present below a new characterization of the reverse-order law.

Theorem 3.11 Let $\mathcal{A} \in \mathbb{C}^{I_{1} \times \cdots \times I_{M} \times J_{1} \times \cdots \times J_{N}}$ and $\mathcal{B} \in \mathbb{C}^{J_{1} \times \cdots \times J_{N} \times K_{1} \times \cdots \times K_{L}}$. Then $\left(\mathcal{A} *_{N} \mathcal{B}\right)^{\dagger}=\mathcal{B}^{\dagger} *_{N} \mathcal{A}^{\dagger}$ is equivalent to

(i) $\left(\mathcal{A} *_{N} \mathcal{A}^{H} *_{M} \mathcal{A} *_{N} \mathcal{B} *_{L} \mathcal{B}^{H} *_{N} \mathcal{B}\right)^{\dagger}=\left(\mathcal{B} *_{L} \mathcal{B}^{H} *_{N} \mathcal{B}\right)^{\dagger} *_{N}\left(\mathcal{A} *_{N} \mathcal{A}^{H} *_{M} \mathcal{A}\right)^{\dagger}$,

(ii) $\left(\mathcal{A} *_{N} \mathcal{B}\right)^{\dagger}=\mathcal{B}^{H} *_{N} \mathcal{B} *_{L}\left(\mathcal{A} *_{N} \mathcal{A}^{H} *_{M} \mathcal{A} *_{N} \mathcal{B} *_{L} \mathcal{B}^{H} *_{N} \mathcal{B}\right)^{\dagger} *_{M} \mathcal{A} *_{N} \mathcal{A}^{H}$.

Proof Consider $\left(\mathcal{A} *_{N} \mathcal{B}\right)^{\dagger}=\mathcal{B}^{\dagger} *_{N} \mathcal{A}^{\dagger}$, so we have $\mathcal{A}^{\dagger} *_{M} \mathcal{A} *_{N} \mathcal{B} *_{L} \mathcal{B}^{H}=\mathcal{B} *_{L} \mathcal{B}^{H} *_{N} \mathcal{A}^{\dagger} *_{M} \mathcal{A}$ and $\mathcal{A}^{H} *_{M} \mathcal{A} *_{N} \mathcal{B} *_{L} \mathcal{B}^{\dagger}=\mathcal{B} *_{L} \mathcal{B}^{\dagger} *_{N} \mathcal{A}^{H} *_{M} \mathcal{A}$. Let $\mathcal{X}=\mathcal{A} *_{N} \mathcal{A}^{H} *_{M} \mathcal{A}$ and $\mathcal{Y}=$ $\mathcal{B} *_{L} \mathcal{B}^{H} *_{N} \mathcal{B}$. From Eqs. (11) and (12), we obtain $\mathcal{X}^{\dagger} *_{M} \mathcal{X}=\mathcal{A}^{\dagger} *_{M} \mathcal{A}$ and $\mathcal{Y} *_{L} \mathcal{Y}^{\dagger}=$ $\mathcal{B} *_{L} B^{\dagger}$. Now, it can be easily seen that $\mathcal{X}^{\dagger} *_{M} \mathcal{X}$ commutes with $\mathcal{Y} *_{L} \mathcal{Y}^{H}$ and $\mathcal{X}^{H} *_{M} \mathcal{X}$ commutes with $\mathcal{Y} *_{L} \mathcal{Y}^{\dagger}$. By Lemma 3.8, we thus have $\left(\mathcal{X}_{*_{N}} \mathcal{Y}\right)^{\dagger}=\mathcal{Y}^{\dagger} *_{N} \mathcal{X}^{\dagger}$, i.e., $\left(\mathcal{A} *_{N} \mathcal{A}^{H} *_{M} \mathcal{A} *_{N} \mathcal{B} *_{L} \mathcal{B}^{H} *_{N} \mathcal{B}\right)^{\dagger}=\left(\mathcal{B} *_{L} \mathcal{B}^{H} *_{N} \mathcal{B}\right)^{\dagger} *_{N}\left(\mathcal{A} *_{N} \mathcal{A}^{H} *_{M} \mathcal{A}\right)^{\dagger}$. Pre-multiplying $\mathcal{B}^{H} *_{N} \mathcal{B}$ and post-multiplying $\mathcal{A} *_{N} \mathcal{A}^{H}$ to (i) yields (ii). Converse part is straightforward.

We have $\left(\mathcal{A}^{H} *_{M} \mathcal{A}\right)^{\dagger}=\mathcal{A}^{\dagger} *_{M} \mathcal{A}^{\dagger H}$ and $\left(\mathcal{A} *_{N} \mathcal{A}^{H}\right)^{\dagger}=\mathcal{A}^{\dagger H} *_{N} \mathcal{A}^{\dagger}$ by Lemma 3.8. Then

$$
\begin{aligned}
{\left[\left(\mathcal{A}^{H} *_{M} \mathcal{A}\right)^{2}\right]^{\dagger} } & =\left[\left(\mathcal{A}^{H} *_{M} \mathcal{A}\right)^{\dagger}\right]^{2} \\
& =\mathcal{A}^{\dagger} *_{M} \mathcal{A}^{\dagger H} *_{N} \mathcal{A}^{\dagger} *_{M} \mathcal{A}^{\dagger H},
\end{aligned}
$$

and

$$
\begin{aligned}
{\left[\left(\mathcal{A} *_{N} \mathcal{A}^{H}\right)^{2}\right]^{\dagger} } & =\left[\left(\mathcal{A} *_{N} \mathcal{A}^{H}\right)^{\dagger}\right]^{2} \\
& =\mathcal{A}^{\dagger H} *_{N} \mathcal{A}^{\dagger} *_{M} \mathcal{A}^{\dagger H} *_{N} \mathcal{A}^{\dagger} .
\end{aligned}
$$

Eqs. (13) and (14) lead the following simpler equivalences.

$$
\begin{aligned}
& \left(\mathcal{A} *_{N} \mathcal{A}^{H}\right)^{2} *_{N}\left[\left(\mathcal{A} *_{N} \mathcal{A}^{H}\right)^{2}\right]^{\dagger}=\mathcal{A} *_{N} \mathcal{A}^{H} *_{M} \mathcal{A} *_{N} \mathcal{A}^{\dagger} *_{M} \mathcal{A} *_{N} \mathcal{A}^{\dagger} *_{M} \mathcal{A}^{\dagger H} *_{N} \mathcal{A}^{\dagger} \\
& =\mathcal{A} *_{N} \mathcal{A}^{\dagger} \text {. }
\end{aligned}
$$

Analogously, one can show that

$$
\begin{aligned}
\left(\mathcal{A}^{H} *_{M} \mathcal{A}\right)^{2} *_{N}\left[\left(\mathcal{A}^{H} *_{M} \mathcal{A}\right)^{2}\right]^{\dagger} & =\mathcal{A}^{\dagger} *_{M} \mathcal{A}, \\
{\left[\left(\mathcal{A}^{H} *_{M} \mathcal{A}\right)^{2}\right]^{\dagger} *_{N}\left(\mathcal{A}^{H} *_{M} \mathcal{A}\right)^{2} } & =\mathcal{A}^{\dagger} *_{M} \mathcal{A}, \\
{\left[\left(\mathcal{A} *_{N} \mathcal{A}^{H}\right)^{2}\right]^{\dagger} *_{N}\left(\mathcal{A} *_{N} \mathcal{A}^{H}\right)^{2} } & =\mathcal{A} *_{N} \mathcal{A}^{\dagger} .
\end{aligned}
$$

We next produce a result that provides another set of equivalent conditions for the reverseorder law using Eqs. (13) to (18).

Theorem 3.12 Let $\mathcal{A} \in \mathbb{C}^{I_{1} \times \cdots \times I_{M} \times J_{1} \times \cdots \times J_{N}}$ and $\mathcal{B} \in \mathbb{C}^{J_{1} \times \cdots \times J_{N} \times K_{1} \times \cdots \times K_{L}}$. Then $\left(\mathcal{A} *_{N} \mathcal{B}\right)^{\dagger}=\mathcal{B}^{\dagger} *_{N} \mathcal{A}^{\dagger}$ is equivalent to

(i) $\left[\left(\mathcal{A}^{H} *_{M} \mathcal{A}\right)^{2} *_{N}\left(\mathcal{B} *_{L} \mathcal{B}^{H}\right)^{2}\right]^{\dagger}=\left[\left(\mathcal{B} *_{L} \mathcal{B}^{H}\right)^{2}\right]^{\dagger} *_{N}\left[\left(\mathcal{A}^{H} *_{M} \mathcal{A}\right)^{2}\right]^{\dagger}$, 
(ii) $\left(\mathcal{A} *_{N} \mathcal{B}\right)^{\dagger}=\mathcal{B}^{H} *_{N} \mathcal{B} *_{L} \mathcal{B}^{H} *_{N}\left[\left(\mathcal{A}^{H} *_{M} \mathcal{A}\right)^{2} *_{N}\left(\mathcal{B} *_{L} \mathcal{B}^{H}\right)^{2}\right]^{\dagger} *_{N} \mathcal{A}^{H} *_{M} \mathcal{A} *_{N} \mathcal{A}^{H}$.

Proof Suppose that $\left(\mathcal{A} *_{N} \mathcal{B}\right)^{\dagger}=\mathcal{B}^{\dagger} *_{N} \mathcal{A}^{\dagger}$, so we have $\mathcal{A}^{\dagger} *_{M} \mathcal{A} *_{N} \mathcal{B} *_{L} \mathcal{B}^{H}=\mathcal{B} *_{L} \mathcal{B}^{H} *_{N} \mathcal{A}^{\dagger} *_{M} \mathcal{A}$ and $\mathcal{A}^{H} *_{M} \mathcal{A} *_{N} \mathcal{B} *_{L} \mathcal{B}^{\dagger}=\mathcal{B} *_{L} \mathcal{B}^{\dagger} *_{N} \mathcal{A}^{H} *_{M} \mathcal{A}$. Let $\mathcal{X}=\left(\mathcal{A}^{H} *_{M} \mathcal{A}\right)^{2}$ and $\mathcal{Y}=\left(\mathcal{B} *_{L} \mathcal{B}^{H}\right)^{2}$. Then using Eqs. (15) and (17), we get $\mathcal{X}^{\dagger} *_{N} \mathcal{X}=\mathcal{A}^{\dagger} *_{M} \mathcal{A}$ and $\mathcal{Y} *_{N} \mathcal{Y}^{\dagger}=\mathcal{B} *_{L} \mathcal{B}^{\dagger}$ which results $\mathcal{X}^{\dagger} *_{N} \mathcal{X} *_{N} \mathcal{Y} *_{N} \mathcal{Y}^{H}=\mathcal{Y} *_{N} \mathcal{Y}^{H} *_{N} \mathcal{X}^{\dagger} *_{N} \mathcal{X}$ and $\mathcal{X}^{H_{*_{N}}} \mathcal{X} *_{N} \mathcal{Y} *_{N} \mathcal{Y}^{\dagger}=$ $\mathcal{Y} *_{N} \mathcal{Y}^{\dagger} *_{N} \mathcal{X}^{H} *_{N} \mathcal{X}$. Thus, $\left(\mathcal{X} *_{N} \mathcal{Y}\right)^{\dagger}=\mathcal{Y}^{\dagger} *_{N} \mathcal{X}^{\dagger}$, i.e., $\left[\left(\mathcal{A}^{H} *_{M} \mathcal{A}\right)^{2} *_{N}\left(\mathcal{B} *_{L} \mathcal{B}^{H}\right)^{2}\right]^{\dagger}=$ $\left[\left(\mathcal{B} *_{L} \mathcal{B}^{H}\right)^{2}\right]^{\dagger} *_{N}\left[\left(\mathcal{A}^{H} *_{M} \mathcal{A}\right)^{2}\right]^{\dagger}$. Pre-multiplying $\mathcal{B}^{H} *_{N} \mathcal{B} *_{L} B^{H}$ and post-multiplying $\mathcal{A}^{H} *_{M} \mathcal{A} *_{N} \mathcal{A}^{H}$ to $(i)$ yields (ii). Converse part is straightforward.

\subsubsection{Reverse-order law using $\{1,2,3\}$-inverse and $\{1,2,4\}$-inverse}

Let us first recall the definitions of a $\{1,2,3\}$-inverse and a $\{1,2,4\}$-inverse of a tensor $\mathcal{A} \in$ $\mathbb{C}^{I_{1} \times \cdots \times I_{M} \times J_{1} \times \cdots \times J_{N}}$. A tensor $\mathcal{X} \in \mathbb{C}^{J_{1} \times \cdots \times J_{N} \times I_{1} \times \cdots \times I_{M}}$ is called a $\{1,2,3\}$-inverse of $\mathcal{A}$ if $\mathcal{X}$ satisfies Eqs. (1) to (3), and is called a $\{1,2,4\}$-inverse of $\mathcal{A}$ if it satisfies Eqs. (1), (2) and (4). Here we obtain some interesting properties of a $\{1,2,3\}$-inverse and a $\{1,2,4\}$-inverse of a tensor which are helpful to deduce some reverse-order law.

Lemma 3.13 Let $\mathcal{X} \in \mathcal{A}\{1,2,3\}$ for any tensor $\mathcal{A} \in \mathbb{C}^{I_{1} \times \cdots \times I_{M} \times J_{1} \times \cdots \times J_{N}}$, then $\mathcal{A} *_{N} \mathcal{X}=$ $\mathcal{A} *_{N} \mathcal{A}^{\dagger}$.

Proof Post-multiplying $\mathcal{X}$ to the fact $\mathcal{A}=\mathcal{A} *_{N} \mathcal{A}^{\dagger} *_{M} \mathcal{A}$, we get $\mathcal{A} *_{N} \mathcal{X}=\mathcal{A} *_{N} \mathcal{A}^{\dagger} *_{M} \mathcal{A} *_{N} \mathcal{X}$. Now, taking conjugate transpose of both sides, we obtain $\mathcal{A} *_{N} \mathcal{X}=\mathcal{A} *_{N} \mathcal{A}^{\dagger}$ due to the fact $\mathcal{X} \in \mathcal{A}\{1,2,3\}$.

Similarly, $\mathcal{X} *_{M} \mathcal{A}=\mathcal{A}^{\dagger} *_{M} \mathcal{A}$ for $\mathcal{X} \in \mathcal{A}\{1,2,4\}$. We next present a characterization of a $\{1,2,3\}$-inverse of a tensor.

Theorem 3.14 Let $\mathcal{A} \in \mathbb{C}^{I_{1} \times \cdots \times I_{M} \times J_{1} \times \cdots \times J_{N}}$. Then $\mathcal{Y}$ is a $\{1,2,3\}$-inverse for $\mathcal{A}$ if and only if there exists a tensor $\mathcal{X} \in \mathbb{C}^{J_{1} \times \cdots \times J_{N} \times I_{1} \times \cdots \times I_{M}}$ such that $\mathcal{A} *_{N} \mathcal{X}=\mathcal{O}, \mathcal{X} *_{M} \mathcal{A} *_{N} \mathcal{A}^{\dagger}=\mathcal{X}$, and $\mathcal{Y}=\mathcal{A}^{\dagger}+\mathcal{X}$.

Proof Let $\mathcal{Y}$ be a $\{1,2,3\}$-inverse for $\mathcal{A}$ and $\mathcal{X}=\mathcal{Y}-\mathcal{A}^{\dagger}$. By Lemma 3.13, we then have $\mathcal{A} *_{N} \mathcal{X}=\mathcal{O}$ and

$$
\begin{aligned}
\mathcal{X} *_{M} \mathcal{A} *_{N} \mathcal{A}^{\dagger} & =\mathcal{Y} *_{M} \mathcal{A} *_{N} \mathcal{A}^{\dagger}-\mathcal{A}^{\dagger} \\
& =\mathcal{Y} *_{M} \mathcal{A} *_{N} \mathcal{Y}-\mathcal{A}^{\dagger} \\
& =\mathcal{X} .
\end{aligned}
$$

Conversely, let $\mathcal{Z}=\mathcal{A}^{\dagger}+\mathcal{X}$. Then

$$
\mathcal{A} *_{N} \mathcal{Z} *_{M} \mathcal{A}=\mathcal{A}
$$

and

$$
\begin{aligned}
\mathcal{Z} *_{M} \mathcal{A} *_{N} \mathcal{Z} & =\mathcal{A}^{\dagger}+\mathcal{X} *_{M} \mathcal{A} *_{N} \mathcal{A}^{\dagger} \\
& =\mathcal{Z} .
\end{aligned}
$$

Also, we have $\mathcal{A} *_{N} \mathcal{Z}=\mathcal{A} *_{N} \mathcal{A}^{\dagger}$. Since $\mathcal{A} *_{N} \mathcal{A}^{\dagger}$ is Hermitian, so $\mathcal{A} *_{N} \mathcal{Z}$ is also Hermitian. Thus, $\mathcal{Y}=\mathcal{Z}=\mathcal{A}^{\dagger}+\mathcal{X}$.

We now present equivalent conditions for the reverse-order law using $\{1,2,3\}$-inverse and $\{1,2,4\}$-inverse. 
Theorem 3.15 Let $\mathcal{A} \in \mathbb{C}^{I_{1} \times \cdots \times I_{M} \times J_{1} \times \cdots \times J_{N}}$ and $\mathcal{B} \in \mathbb{C}^{J_{1} \times \cdots \times J_{N} \times K_{1} \times \cdots \times K_{L}}$. Then $\left(\mathcal{A} *_{N} \mathcal{B}\right)^{\dagger}=\mathcal{B}^{\dagger} *_{N} \mathcal{A}^{\dagger}$ if and only if there exists a $\{1,2,3\}$-inverse $\mathcal{Y}$ of $\mathcal{B}$ satisfying $\left(\mathcal{A} *_{N} \mathcal{B}\right)^{\dagger}=\mathcal{Y} *_{N} \mathcal{A}^{\dagger}$.

Proof Suppose that there exists a $\{1,2,3\}$-inverse $\mathcal{Y}$ such that $\left(\mathcal{A} *_{N} \mathcal{B}\right)^{\dagger}=\mathcal{Y} *_{N} \mathcal{A}^{\dagger}$. By Lemma 2.3, we have $\left(\mathcal{A} *_{N} \mathcal{B}\right)^{\dagger}=\mathcal{B}^{\dagger} *_{N} \mathcal{B} *_{L}\left(\mathcal{A} *_{N} \mathcal{B}\right)^{\dagger}$, whose right-hand side reduces to $\mathcal{B}^{\dagger} *_{N} \mathcal{A}^{\dagger}$ using $\left(\mathcal{A} *_{N} \mathcal{B}\right)^{\dagger}=\mathcal{Y} *_{N} \mathcal{A}^{\dagger}$ and Lemma 3.13. Conversely, since $\mathcal{B}^{\dagger}$ is also a $\{1,2,3\}$ inverse of $\mathcal{B}$, so the claim is justified.

Analogously, one can prove that $\left(\mathcal{A} *_{N} \mathcal{B}\right)^{\dagger}=\mathcal{B}^{\dagger} *_{N} \mathcal{A}^{\dagger}$ if and only if there exists a $\{1,2,4\}-$ inverse $\mathcal{Y}$ of $\mathcal{A}$ satisfying $\left(\mathcal{A} *_{N} \mathcal{B}\right)^{\dagger}=\mathcal{B}^{\dagger} *_{N} \mathcal{Y}$.

\subsection{Application to additive results on the Moore-Penrose inverse}

As an application of the reverse-order law, we have the following result.

Theorem 3.16 Let $\mathcal{A}$ be any tensor and $\mathcal{V}$ be a Hermitian tensor such that $\mathcal{A}^{\dagger} *_{M} \mathcal{A} *_{N} \mathcal{V}=\mathcal{V}$

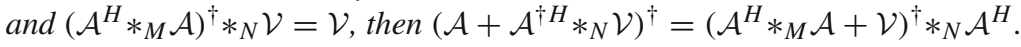

Proof In order to proof this, we make use of Theorem 2.4. Observe that $\mathcal{A}+\mathcal{A}^{\dagger H_{*}} \mathcal{V}=$ $\mathcal{A}^{\dagger H} *_{N}\left(\mathcal{A}^{H} *_{M} \mathcal{A}+\mathcal{V}\right)$. Let $\mathcal{X}=\mathcal{A}^{\dagger H}$ and $\mathcal{Y}=\mathcal{A}^{H} *_{M} \mathcal{A}+\mathcal{V}$. By taking the conjugate transpose of $\mathcal{A}^{\dagger} *_{M} \mathcal{A} *_{N} \mathcal{V}=\mathcal{V}$ we get $\mathcal{V} *_{N} \mathcal{A}^{\dagger} *_{M} \mathcal{A}=\mathcal{V}$. With these results in hand, it is easy to show that $\mathcal{X}^{\dagger} *_{M} \mathcal{X} *_{N} \mathcal{Y}=\mathcal{Y} *_{N} \mathcal{X}^{\dagger} *_{M} \mathcal{X}$. Thus, $\mathcal{X}^{\dagger} *_{M} \mathcal{X}$ commutes with $\mathcal{Y} *_{N} \mathcal{Y}^{H}$. So, $\mathcal{X}^{\dagger} *_{M} \mathcal{X} *_{N} \mathcal{Y} *_{N} \mathcal{Y}^{H} *_{N} \mathcal{X}^{H}=\mathcal{Y} *_{N} \mathcal{Y}^{H} *_{N} \mathcal{X}^{H}$.

Also, notice that the hypothesis $\left(\mathcal{A}^{H} *_{M} \mathcal{A}\right)^{\dagger} *_{N} \mathcal{V}=\mathcal{V}$ implies $\mathcal{V} *_{N}\left(\mathcal{A}^{H} *_{M} \mathcal{A}\right)^{\dagger}=\mathcal{V}$ and hence $\mathcal{X}^{H} *_{M} \mathcal{X} *_{N} \mathcal{Y}=\mathcal{Y} *_{N} \mathcal{X}^{H} *_{M} \mathcal{X}$. Thus, we have

$$
\mathcal{Y} *_{N} \mathcal{Y}^{\dagger} *_{N} \mathcal{X}^{H} *_{M} \mathcal{X} *_{N} \mathcal{Y}=\mathcal{X}^{H} *_{M} \mathcal{X}_{*_{N}} \mathcal{Y}
$$

Hence, the result follows by Theorem 2.4 .

Analogously, it can be proved that for a Hermitian tensor $\mathcal{V}$ such that $\mathcal{A} *_{N} \mathcal{A}^{\dagger} *_{M} \mathcal{V}=\mathcal{V}$ and $\left(\mathcal{A} *_{N} \mathcal{A}^{H}\right)^{\dagger} *_{M} \mathcal{V}=\mathcal{V}$, we have $\left(\mathcal{A}+\mathcal{V} *_{M} \mathcal{A}^{\dagger H}\right)^{\dagger}=\mathcal{A}^{H} *_{M}\left(\mathcal{A} *_{N} \mathcal{A}^{H}+\mathcal{V}\right)^{\dagger}$. The above facts are motivated from [28] where the authors considered the solution of linear equations of the type $A x=b+\epsilon$ where $A$ is an $m \times n$ matrix of rank $r$ (with $r \leq m \leq n$ ), $x$ is a column vector of order $n$ and both $b$ and $\epsilon$ are column vectors of order $m$. Such types of problems occur in the remote sounding of atmospheric temperature profile by satellites (see [28] and the references cited therein for more details). The above two problems also lead to a more general problem, i.e., finding the Moore-Penrose inverse of the sum of two tensors, and the next two results are in this direction. Further study on this is left for future study.

Theorem 3.17 Let $\mathcal{A}$ and $\mathcal{B}$ be arbitrary tensors such that $\mathcal{I}+\mathcal{A}^{\dagger} *_{M} \mathcal{B}$ is invertible and satisfies the following

$$
\begin{aligned}
& \mathcal{A} *_{N} \mathcal{A}^{\dagger} *_{M} \mathcal{B}=\mathcal{B}, \\
& \mathcal{B} *_{N} \mathcal{A}^{\dagger} *_{{ }_{M}} \mathcal{A}=\mathcal{B},
\end{aligned}
$$

then

$$
(\mathcal{A}+\mathcal{B})^{\dagger}=\left(I+\mathcal{A}^{\dagger} *_{M} \mathcal{B}\right)^{-1} *_{N} \mathcal{A}^{\dagger}
$$


Proof One can quickly see $\mathcal{A}+\mathcal{B}=\mathcal{A} *_{N}\left(\mathcal{I}+\mathcal{A}^{\dagger} *_{M} \mathcal{B}\right)$. It is therefore enough to prove that $\mathcal{A} *_{N}\left(\mathcal{I}+\mathcal{A}^{\dagger} *_{M} \mathcal{B}\right)$ follows the reverse-order law in order to assert our hypothesis. Now, we have

$$
\mathcal{A}^{\dagger} *_{M} \mathcal{A} *_{N}\left(\mathcal{I}+\mathcal{A}^{\dagger} *_{M} \mathcal{B}\right) *_{N}\left(\mathcal{I}+\mathcal{A}^{\dagger} *_{M} \mathcal{B}\right)^{H} *_{N} \mathcal{A}^{H}=\mathcal{A}^{\dagger} *_{M} \mathcal{A} *_{N}\left(\mathcal{I}+\mathcal{A}^{\dagger} *_{M} \mathcal{B}\right) *_{N}\left(\mathcal{A}^{H}+\mathcal{B}^{H}\right)
$$

because of Eq. (19). Again, using Eq. (20), and the fact that $\mathcal{A}^{\dagger} *_{M} \mathcal{A} *_{N} \mathcal{A}^{H}=\mathcal{A}^{H}$ and $\mathcal{A}^{\dagger} *_{M} \mathcal{A} *_{N} \mathcal{A}^{\dagger}=\mathcal{A}^{\dagger}$ in the left hand side of the Eq. (21), we get

$$
\mathcal{A}^{\dagger} *_{M} \mathcal{A} *_{N}\left(\mathcal{I}+\mathcal{A}^{\dagger} *_{M} \mathcal{B}\right) *_{N}\left(\mathcal{I}+\mathcal{A}^{\dagger} *_{M} \mathcal{B}\right){ }^{H} *_{N} \mathcal{A}^{H}=\left(\mathcal{I}+\mathcal{A}^{\dagger} *_{M} \mathcal{B}\right) *_{N}\left(\mathcal{I}+\mathcal{A}^{\dagger} *_{M} \mathcal{B}\right){ }^{H} *_{N} \mathcal{A}^{H},
$$

and since by assumption $\mathcal{I}+\mathcal{A}^{\dagger} *_{M} \mathcal{B}$ is invertible, so we have

$$
\mathcal{A} *_{N}\left(\mathcal{I}+\mathcal{A}^{\dagger} *_{M} \mathcal{B}\right) *_{N}\left(\mathcal{I}+\mathcal{A}^{\dagger} *_{M} \mathcal{B}\right)^{\dagger} *_{N} \mathcal{A}^{\dagger}=\mathcal{A} *_{N} \mathcal{A}^{\dagger}
$$

The argument is therefore accompanied by Theorem 3.6.

We have got the following finding likewise.

Theorem 3.18 Let $\mathcal{A}$ and $\mathcal{B}$ be arbitrary tensors such that $\mathcal{I}-\mathcal{A}^{\dagger} *_{M} \mathcal{B}$ is invertible and satisfy the following

$$
\begin{aligned}
& \mathcal{A} *_{N} \mathcal{A}^{\dagger} *_{M} \mathcal{B}=\mathcal{B}, \\
& \mathcal{B} *_{N} \mathcal{A}^{\dagger} *_{M} \mathcal{A}=\mathcal{B},
\end{aligned}
$$

then

$$
(\mathcal{A}-\mathcal{B})^{\dagger}=\left(I-\mathcal{A}^{\dagger} *_{M} \mathcal{B}\right)^{-1} *_{N} \mathcal{A}^{\dagger} .
$$

The conditions $\mathcal{I}+\mathcal{A}^{\dagger} *_{M} \mathcal{B}$ and $\mathcal{I}-\mathcal{A}^{\dagger} *_{M} \mathcal{B}$ are invertible can be relaxed by using the notion of eigenvalue of tensor. This is recalled next from the very recent work by Liang and Zheng [19] which appeared in 2019.

Definition 3.19 (Definition 2.3, [19]). Let $\mathcal{A} \in \mathbb{C}^{I_{1} \times \cdots \times I_{N} \times I_{1} \times \cdots \times I_{N}}$ be a given tensor. If a complex number $\lambda$ and a nonzero tensor $\mathcal{X} \in \mathbb{C}^{I_{1} \times \cdots \times I_{N}}$ satisfy $\mathcal{A} *_{N} \mathcal{X}=\lambda \mathcal{X}$.

The spectrum of a tensor $\mathcal{A}$ is the set of all eigenvalues of a tensor $\mathcal{A}$ and is denoted by $\Omega(\mathcal{A})$, i.e.,

$$
\Omega(\mathcal{A})=\left\{\lambda: \mathcal{A} *_{N} \mathcal{X}=\lambda \mathcal{X}, \mathcal{X} \neq 0\right\} .
$$

The invertibilty of a tensor is confirmed through its eigenvalue, and is shown next.

Lemma 3.20 A tensor is invertible if and only if all its eigenvalues are nonzero.

Proof Let $\mathcal{A} \in \mathbb{C}^{I_{1} \times \cdots \times I_{N} \times I_{1} \times \cdots \times I_{N}}$ be an invertible tensor. Let 0 be an eigenvalue and $\mathcal{X}(\neq \mathcal{O}) \in \mathbb{C}^{I_{1} \times \cdots \times I_{N}}$ is an eigentensor corresponding to the eigenvalue 0 . Then

$$
\mathcal{A} *_{N} \mathcal{X}=0 \mathcal{X}=\mathcal{O}
$$

Now, on pre-multiplying Eq. (24) with $\mathcal{A}^{-1}$ we get $\mathcal{X}=\mathcal{O}$, which is a contradiction. Thus, 0 cannot be an eigenvalue of an invertible tensor $\mathcal{A}$.

Conversely, suppose that all the eigenvalues of a tensor $\mathcal{A}$ are nonzero. Let the tensor $\mathcal{A}$ be not invertible, then there exists a nonzero tensor $\mathcal{X} \in \mathbb{C}^{I_{1} \times \cdots \times I_{N}}$ such that $\mathcal{A} *_{N} \mathcal{X}=\mathcal{O}=0 \mathcal{X}$. But, this implies 0 is an eigenvalue of $\mathcal{A}$, which is a contradiction. Thus, $\mathcal{A}$ is invertible. 
The relation between the eigenvalues and eigentensors of tensors $\mathcal{A}$ and $\mathcal{I}-\mathcal{A}$ is explained next. Since $\lambda$ is an eigenvalue of a tensor $\mathcal{A} \in \mathbb{C}^{I_{1} \times \cdots \times I_{N} \times I_{1} \times \cdots \times I_{N}}$ corresponding to eigentensor $\mathcal{X}$, so we have

$$
\mathcal{A} *_{N} \mathcal{X}=\lambda \mathcal{X}
$$

Thus,

$$
(\mathcal{I}-\mathcal{A}) *_{N} \mathcal{X}=(1-\lambda) \mathcal{X}
$$

Hence, if $\lambda$ is an eigenvalue of a tensor $\mathcal{A} \in \mathbb{C}^{I_{1} \times \cdots \times I_{N} \times I_{1} \times \cdots \times I_{N}}$ corresponding to eigentensor $\mathcal{X}$, then $1-\lambda$ is an eigenvalue of $\mathcal{I}-\mathcal{A}$ with the same eigentensor. Similarly, if $\lambda$ is an eigenvalue of a tensor $\mathcal{A} \in \mathbb{C}^{I_{1} \times \cdots \times I_{N} \times I_{1} \times \cdots \times I_{N}}$ corresponding to eigentensor $\mathcal{X}$, then $1+\lambda$ is an eigenvalue of $\mathcal{I}+\mathcal{A}$ with the same eigentensor. Based on the above discussion, we have the following lemma.

Lemma 3.21 If $1 \notin \Omega(\mathcal{A})$, then $\mathcal{I}-\mathcal{A}$ is invertible.

Thus, Theorem 3.18 can be restated as following.

Theorem 3.22 Let $\mathcal{A}$ and $\mathcal{B}$ be arbitrary tensors such that $1 \notin \Omega\left(\mathcal{A}^{\dagger} *_{M} \mathcal{B}\right)$ and satisfy the following

$$
\begin{aligned}
& \mathcal{A} *_{N} \mathcal{A}^{\dagger} *_{M} \mathcal{B}=\mathcal{B}, \\
& \mathcal{B} *_{N} \mathcal{A}^{\dagger} *_{M} \mathcal{A}=\mathcal{B},
\end{aligned}
$$

then

$$
(\mathcal{A}-\mathcal{B})^{\dagger}=\left(I-\mathcal{A}^{\dagger} *_{M} \mathcal{B}\right)^{-1} *_{N} \mathcal{A}^{\dagger}
$$

Motivated by the above results, we next consider the problem of computing bounds of $\left\|\mathcal{B}^{\dagger}-\mathcal{A}^{\dagger}\right\|_{F}$ which is known as Additive perturbation bounds of the Moore-Penrose inverse. To do this, we recall the following two norms and investigate some of their properties in tensor space to explore the additive perturbation bounds of the Moore-Penrose inverse.

Definition 3.23 A tensor norm $\|\cdot\|$ defined on a multilinear space $I_{1} \times \cdots \times I_{M} \times J_{1} \times \cdots \times J_{N}$ is said to be unitarily invariant if for each $\mathcal{A} \in \mathbb{C}^{I_{1} \times \cdots \times I_{M} \times J_{1} \times \cdots \times J_{N}}$ and unitary tensor $\mathcal{U} \in \mathbb{C}^{I_{1} \times \cdots \times I_{M} \times I_{1} \times \cdots \times I_{M}}$ and $\mathcal{V} \in \mathbb{C}^{J_{1} \times \cdots \times J_{N} \times J_{1} \times \cdots \times J_{N}}$

$$
\left\|\mathcal{U} *_{M} \mathcal{A} *_{N} \mathcal{V}\right\|=\|\mathcal{A}\| .
$$

Recently, Ma et al. [20] defined the spectral norm of even-order square tensor as follows

Definition 3.24 (Lemma 2.1, [20]). The spectral norm of a tensor $\mathcal{A} \in \mathbb{C}^{I_{1} \times \cdots \times I_{M} \times J_{1} \times \cdots \times J_{N}}$ is defined as the square root of maximum eigenvalue of $\mathcal{A}^{H} *_{M} \mathcal{A}$ and is denoted by $\|\mathcal{A}\|_{2}$, i.e.,

$$
\begin{aligned}
\|\mathcal{A}\|_{2} & =\left(\text { maximum eigenvalue of } \mathcal{A}^{H} *_{M} \mathcal{A}\right)^{1 / 2} \\
& =\text { largest singular value of } \mathcal{A} .
\end{aligned}
$$

Ma et al. [20] proved that the spectral norm of the Moore-Penrose inverse of a tensor is equal to the reciprocal of smallest nonzero singular value of that tensor. The same result for arbitrary order tensor is stated next, and for the proof, one may follow the same steps as in Lemma 3.2, [20]. 
Lemma 3.25 Let $\mathcal{A} \in \mathbb{C}^{I_{1} \times \cdots \times I_{M} \times J_{1} \times \cdots \times J_{N}}$. Then

$$
\begin{aligned}
\|\mathcal{A}\|_{2} & =\text { largest singular value of } \mathcal{A} \text {, and } \\
\left\|\mathcal{A}^{\dagger}\right\|_{2} & =\frac{1}{\text { smallest nonzero singular value of } \mathcal{A}} .
\end{aligned}
$$

Further, note that the Frobenius norm $\|.\|_{F}$ of $\mathcal{A} \in \mathbb{C}^{I_{1} \times \cdots \times I_{M} \times J_{1} \times \cdots \times J_{N}}$ is defined ( [26]) as

$$
\begin{aligned}
\|\mathcal{A}\|_{F} & =\left(\operatorname{trace}\left(\mathcal{A}^{H} *_{M} \mathcal{A}\right)\right)^{1 / 2} \\
& =\left(\sum_{i_{1} \cdots i_{N} j_{1} \cdots j_{N}}\left|a_{i_{1} \cdots i_{N} j_{1} \cdots j_{N}}\right|^{2}\right)^{1 / 2} .
\end{aligned}
$$

We now have the following results for the aforementioned Frobenius norm.

Lemma 3.26 The Frobenius norm is unitarily invariant.

Proof The claim is equivalent to show that for $\mathcal{A} \in \mathbb{C}^{I_{1} \times \cdots \times I_{M} \times J_{1} \times \cdots \times J_{N}}$,

$$
\left\|\mathcal{U} *_{M} \mathcal{A} *_{N} \mathcal{V}\right\|_{F}=\|\mathcal{A}\|_{F},
$$

where $\mathcal{U} \in \mathbb{C}^{I_{1} \times \cdots \times I_{M} \times I_{1} \times \cdots \times I_{M}}$ and $\mathcal{V} \in \mathbb{C}^{J_{1} \times \cdots \times J_{N} \times J_{1} \times \cdots \times J_{N}}$ are any two unitary tensors. Using the definition of the Frobenius norm, we have

$$
\begin{aligned}
\left\|\mathcal{U} *_{M} \mathcal{A} *_{N} \mathcal{V}\right\|_{F} & =\left(\operatorname{trace}\left(\left(\mathcal{U} *_{M} \mathcal{A} *_{N} \mathcal{V}\right)^{H} *_{M}\left(\mathcal{U} *_{M} \mathcal{A} *_{N} \mathcal{V}\right)\right)\right)^{1 / 2} \\
& =\left(\operatorname{trace}\left(\mathcal{V}^{H} *_{N} \mathcal{A}^{H} *_{M} \mathcal{A} *_{N} \mathcal{V}\right)\right)^{1 / 2} \\
& =\left(\operatorname{trace}\left(\mathcal{V}^{H} *_{N} \mathcal{V} *_{N} \mathcal{A}^{H} *_{M} \mathcal{A}\right)\right)^{1 / 2} \\
& =\left(\operatorname{trace}\left(\mathcal{A}^{H} *_{M} \mathcal{A}\right)\right)^{1 / 2} \\
& =\|\mathcal{A}\|_{F} .
\end{aligned}
$$

Lemma 3.27 Let $\mathcal{U}=\left[\mathcal{U}_{1} \mathcal{U}_{2}\right] \in \mathbb{C}^{I_{1} \times \cdots \times I_{M} \times I_{1} \times \cdots \times I_{M}}$ and $\mathcal{V}=\left[\mathcal{V}_{1} \mathcal{V}_{2}\right] \in \mathbb{C}^{J_{1} \times \cdots \times J_{N} \times J_{1} \times \cdots \times J_{N}}$ be two unitary tensors. Then,

$\|\mathcal{E}\|_{F}^{2}=\left\|\mathcal{U}_{1}^{H} *_{M} \mathcal{E} *_{N} \mathcal{V}_{1}\right\|_{F}^{2}+\left\|\mathcal{U}_{1}^{H} *_{M} \mathcal{E} *_{N} \mathcal{V}_{2}\right\|_{F}^{2}+\left\|\mathcal{U}_{2}^{H} *_{M} \mathcal{E} *_{N} \mathcal{V}_{1}\right\|_{F}^{2}+\left\|\mathcal{U}_{2}^{H} *_{M} \mathcal{E} *_{N} \mathcal{V}_{2}\right\|_{F}^{2}$,

for any tensor $\mathcal{E} \in \mathbb{C}^{I_{1} \times \cdots \times I_{M} \times J_{1} \times \cdots \times J_{N}}$.

Proof Since the Frobenius norm is unitarily invariant, so we have

$$
\begin{aligned}
\|\mathcal{E}\|_{F}^{2} & =\left\|\mathcal{U}^{H} *_{M} \mathcal{E} *_{N} \mathcal{V}\right\|_{F}^{2} \\
& =\left\|\left[\begin{array}{c}
U_{1}^{H} \\
\mathcal{U}_{2}^{H}
\end{array}\right] *_{M} \mathcal{E} *_{N}\left[\begin{array}{ll}
\mathcal{V}_{1} & \mathcal{V}_{2}
\end{array}\right]\right\|_{F}^{2} \\
& =\left\|\left[\begin{array}{l}
\mathcal{U}_{1}^{H} *_{M} \mathcal{E} *_{N} \mathcal{V}_{1} \mathcal{U}_{1}^{H} *_{M} \mathcal{E} *_{N} \mathcal{V}_{2} \\
\mathcal{U}_{2}^{H} *_{M} \mathcal{E} *_{N} \mathcal{V}_{1} \mathcal{U}_{2}^{H} *_{M} \mathcal{E} *_{N} \mathcal{V}_{2}
\end{array}\right]\right\|_{F}^{2} \\
& =\left\|\mathcal{U}_{1}^{H} *_{M} \mathcal{E} *_{N} \mathcal{V}_{1}\right\|_{F}^{2}+\left\|\mathcal{U}_{1}^{H} *_{M} \mathcal{E} *_{N} \mathcal{V}_{2}\right\|_{F}^{2}+\left\|\mathcal{U}_{2}^{H} *_{M} \mathcal{E} *_{N} \mathcal{V}_{1}\right\|_{F}^{2}+\left\|\mathcal{U}_{2}^{H} *_{M} \mathcal{E} *_{N} \mathcal{V}_{2}\right\|_{F}^{2} .
\end{aligned}
$$


Let $\mathcal{A}, \mathcal{E} \in \mathbb{C}^{I_{1} \times \cdots \times I_{N} \times J_{1} \times \cdots \times J_{N}}$ and $\mathcal{B}=\mathcal{A}+\mathcal{E}$. Let $\mathcal{A}=\mathcal{U} *_{M} \mathcal{D} *_{N} \mathcal{V}^{H}$ and $\mathcal{B}=\mathcal{R} *{ }_{M} \mathcal{S} *_{N} \mathcal{T}^{H}$ be the singular value decompositions (SVD) of $\mathcal{A}$ and $\mathcal{B}$, where $\mathcal{U}, \mathcal{R} \in \mathbb{C}^{I_{1} \times \cdots \times I_{N} \times I_{1} \times \cdots \times I_{N}}$ and $\mathcal{V}, \mathcal{T} \in \mathbb{C}^{J_{1} \times \cdots \times J_{N} \times J_{1} \times \cdots \times J_{N}}$ satisfy

$$
\mathcal{U} *_{N} \mathcal{U}^{H}=\mathcal{I}_{N}, \mathcal{V} *_{N} \mathcal{V}=\mathcal{I}_{N}, \mathcal{R} *_{N} \mathcal{R}^{H}=\mathcal{I}_{N}, \mathcal{T} *_{N} \mathcal{T}^{H}=\mathcal{I}_{N} .
$$

Suppose that $\operatorname{rank}_{U}(\mathcal{A})=r$ and $\operatorname{rank}_{U}(\mathcal{B})=s$. Let us arrange the tensors involving in the $\mathrm{SVD}$ of $\mathcal{A}$ and $\mathcal{B}$ as the following block tensors.

$$
\begin{aligned}
& \mathcal{U}=\left[\begin{array}{ll}
\mathcal{U}_{1} & \mathcal{U}_{2}
\end{array}\right], \quad \mathcal{D}=\left[\begin{array}{cc}
\mathcal{D}_{1} & \mathcal{O} \\
\mathcal{O} & \mathcal{O}
\end{array}\right], \mathcal{V}=\left[\begin{array}{ll}
\mathcal{V}_{1} & \mathcal{V}_{2}
\end{array}\right] \\
& \mathcal{R}=\left[\begin{array}{ll}
\mathcal{R}_{1} & \mathcal{R}_{2}
\end{array}\right], \mathcal{S}=\left[\begin{array}{ll}
\mathcal{S}_{1} & \mathcal{O} \\
\mathcal{O} & \mathcal{O}
\end{array}\right], \mathcal{T}=\left[\begin{array}{ll}
\mathcal{T}_{1} & \mathcal{T}_{2}
\end{array}\right],
\end{aligned}
$$

where $\mathcal{U}_{1} \in \mathbb{C}^{I_{1} \times \cdots \times I_{N} \times K_{1} \times \cdots \times K_{N}}, \mathcal{U}_{2} \in \mathbb{C}^{I_{1} \times \cdots \times I_{N} \times L_{1} \times \cdots \times L_{M}}$, such that $K_{i}+L_{i}=I_{i}$ for $i=1,2, \cdots, N, \mathcal{V}_{1} \in \mathbb{C}^{J_{1} \times \cdots \times J_{N} \times K_{1} \times \cdots \times K_{N}}, \mathcal{V}_{2} \in \mathbb{C}^{J_{1} \times \cdots \times J_{N} \times Q_{1} \times \cdots \times Q_{N}}$ such that $K_{i}+$ $Q_{i}=J_{i}$ for $i=1,2, \cdots, N ; \mathcal{R}_{1} \in \mathbb{C}^{I_{1} \times \cdots \times I_{N} \times \tilde{K}_{1} \times \cdots \times \tilde{K}_{N}}, \mathcal{R}_{2} \in \mathbb{C}^{I_{1} \times \cdots \times I_{N} \times \tilde{L}_{1} \times \cdots \times \tilde{L}_{N}}$, such that $\tilde{K}_{i}+\tilde{L}_{i}=I_{i}$ for $i=1,2, \ldots, N, \mathcal{T}_{1} \in \mathbb{C}^{J_{1} \times \cdots \times J_{N} \times \tilde{K}_{1} \times \cdots \times \tilde{K}_{N}}, \mathcal{T}_{2} \in$ $\mathbb{C}^{J_{1} \times \cdots \times J_{N} \times \tilde{Q}_{1} \times \cdots \times \tilde{Q}_{N}}$ such that $\tilde{K}_{i}+\tilde{Q}_{i}=J_{i}$ for $i=1,2, \ldots, N$. Here $\mathcal{D}_{1} \in$ $\mathbb{C}^{K_{1} \times \cdots \times K_{N} \times K_{1} \times \cdots \times K_{N}}$ and $\mathcal{S}_{1} \in \mathbb{C}^{\tilde{K}_{1} \times \cdots \times \tilde{K}_{N} \times \tilde{K}_{1} \times \cdots \times \tilde{K}_{N}}$ with $K_{1} \cdot \ldots \cdot K_{N}=r$ and $\tilde{K}_{1} \cdot \ldots \cdot \tilde{K}_{N}=s$ are diagonal tensors defined element-wise as follow

$$
\left(\mathcal{D}_{1}\right)_{k_{1} \cdots k_{N} j_{1} \cdots j_{N}}= \begin{cases}\sigma_{i \operatorname{vec}(\mathbf{k}, \mathbb{K})}, & \text { if } i \operatorname{vec}(\mathbf{k}, \mathbb{K})=\operatorname{ivec}(\mathbf{j}, \mathbb{J}), \\ 0, & \text { otherwise }\end{cases}
$$

and

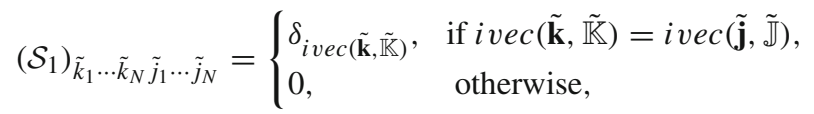

where $\mathbf{k}:=\left\{k_{1}, \ldots, k_{N}\right\}, \mathbf{j}:=\left\{j_{1}, \ldots, j_{N}\right\}, \tilde{\mathbf{k}}:=\left\{\tilde{k}_{1}, \ldots, \tilde{k}_{M}\right\}, \tilde{\mathbf{j}}:=\left\{\tilde{j}_{1}, \ldots, \tilde{j}_{N}\right\}$,

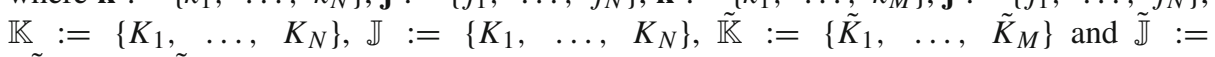
$\left\{\tilde{K}_{1}, \ldots, \tilde{K}_{M}\right\} ; \sigma_{1} \geq \sigma_{2} \geq \cdots \geq \sigma_{r}>0$ and $\delta_{1} \geq \delta_{2} \geq \cdots \geq \delta_{s}>0$ are singular values of $\mathcal{A}$ and $\mathcal{B}$, respectively. By Proposition 2.4 [26], we then have

$$
\mathcal{A}=\mathcal{U}_{1} *_{N} \mathcal{D}_{1} *_{N} \mathcal{V}_{1}^{H}
$$

and

$$
\mathcal{B}=\mathcal{R}_{1} *_{N} \mathcal{S}_{1} *_{N} \mathcal{T}_{1}^{H}
$$

So,

$$
\begin{aligned}
\mathcal{E} & =\mathcal{B}-\mathcal{A} \\
& =R_{1} *_{N} \mathcal{S}_{1} *_{N} \mathcal{T}_{1}^{H}-\mathcal{U}_{1} *_{N} \mathcal{D}_{1} *_{N} \mathcal{V}_{1}^{H}
\end{aligned}
$$

Thus,

$$
\mathcal{R}_{1}^{H} *_{N} \mathcal{E} *_{N} \mathcal{V}_{1}=\mathcal{S}_{1} *_{N} \mathcal{T}^{H} *_{N} \mathcal{V}_{1}-\mathcal{R}_{1}^{H} *_{N} \mathcal{U}_{1} *_{N} \mathcal{D}_{1}
$$

and

$$
\mathcal{U}_{1}^{H} *_{N} \mathcal{E} *_{N} \mathcal{T}_{1}=\mathcal{U}_{1}^{H} *_{N} \mathcal{R}_{1} *_{N} \mathcal{S}_{1}-\mathcal{D}_{1} *_{N} \mathcal{V}_{1}^{H} *_{N} \mathcal{T}_{1}
$$


From Eqs. (30) and (31), we now obtain

$$
\mathcal{S}_{1}^{-1} *_{N} \mathcal{R}_{1}^{H} *_{N} \mathcal{E} *_{N} \mathcal{V}_{1} *_{N} \mathcal{D}_{1}^{-1}=\mathcal{T}^{H} *_{N} \mathcal{V}_{1} *_{N} \mathcal{D}_{1}^{-1}-\mathcal{S}_{1}^{-1} *_{N} \mathcal{R}_{1}^{H} *_{N} \mathcal{U}_{1}
$$

and

$$
\mathcal{D}_{1}^{-1} *_{N} \mathcal{U}_{1}^{H} *_{N} \mathcal{E} *_{N} \mathcal{T}_{1} *_{N} \mathcal{S}_{1}^{-1}=\mathcal{D}_{1}^{-1} *_{N} \mathcal{U}_{1}^{H} *_{N} \mathcal{R}_{1}-\mathcal{V}_{1}^{H} *_{N} \mathcal{T}_{1} *_{N} \mathcal{S}_{1}^{-1}
$$

Since $\mathcal{U}_{2}^{H}{ }{ }_{N} \mathcal{U}_{1}=\mathcal{O}$ and $\mathcal{T}_{1}{ }^{H}{ }_{N} \mathcal{T}_{2}=\mathcal{O}$, we get

$$
\mathcal{U}_{2}^{H} *_{N} \mathcal{E} *_{N} \mathcal{T}_{1}=\mathcal{U}_{2}^{H} *_{N} \mathcal{R}_{1} *_{N} \mathcal{S}_{1}
$$

and

$$
\mathcal{U}_{1}^{H} *_{N} \mathcal{E} *_{N} \mathcal{T}_{2}=-\mathcal{D}_{1} *_{N} \mathcal{V}_{1}^{H} *_{N} \mathcal{T}_{2}
$$

Since $\mathcal{V}_{1}^{H} *_{N} \mathcal{V}_{2}=\mathcal{O}$ and $\mathcal{R}_{2}^{H} *_{N} \mathcal{R}_{1}=\mathcal{O}$, so

$$
\mathcal{R}_{1}^{H} *_{N} \mathcal{E} *_{N} \mathcal{V}_{2}=\mathcal{S}_{1} *_{N} \mathcal{T}_{1}^{H} *_{N} \mathcal{V}_{2}
$$

and

$$
\mathcal{R}_{2}^{H} *_{N} \mathcal{E} *_{N} \mathcal{V}_{1}=-\mathcal{R}_{2}^{H} *_{N} \mathcal{U}_{1} *_{N} \mathcal{D}_{1}
$$

Also, we have

$$
\begin{aligned}
\mathcal{S}_{1}^{-1} *_{N} \mathcal{R}_{1}^{H} *_{N} \mathcal{U}_{2} & =\mathcal{S}_{1}^{-2} *_{N}\left(\mathcal{U}_{2}^{H} *_{N} \mathcal{R}_{1} *_{N} \mathcal{S}_{1}\right)^{H} \\
& =\mathcal{S}_{1}^{-2} *_{N}\left(\mathcal{U}_{2}^{H} *_{N} \mathcal{E}_{*_{N}} \mathcal{T}_{1}\right)^{H}
\end{aligned}
$$

and

$$
\begin{aligned}
-\mathcal{T}_{2}^{H} *_{N} \mathcal{V}_{1} *_{N} \mathcal{D}_{1}^{-1} & =\left(-\mathcal{D}_{1} *_{N} \mathcal{V}_{1}^{H} *_{N} \mathcal{T}_{2}\right)^{H} *_{N} \mathcal{D}_{1}^{-2} \\
& =\left(\mathcal{U}_{1}^{H} *_{N} \mathcal{E} *_{N} \mathcal{T}_{2}\right)^{H} *_{N} \mathcal{D}_{1}^{-2}
\end{aligned}
$$

The additive perturbation bounds for the Moore-Penrose inverse of arbitrary order tensors via the Einstein product by means of the Frobenius norm is obtained next.

Theorem 3.28 Let $\mathcal{A}, \mathcal{E} \in \mathbb{C}^{I_{1} \times \cdots \times I_{N} \times J_{1} \times \cdots \times J_{N}}$ and $\mathcal{B}=\mathcal{A}+\mathcal{E}$. If $\operatorname{rank}_{U}(\mathcal{A})=r$ and $\operatorname{rank}_{U}(\mathcal{B})=s$, then

$$
\left\|\mathcal{B}^{\dagger}-\mathcal{A}^{\dagger}\right\|_{F} \leq \max \left\{\left\|\mathcal{A}^{\dagger}\right\|_{2}\left\|\mathcal{B}^{\dagger}\right\|_{2},\left\|\mathcal{A}^{\dagger}\right\|_{2}^{2},\left\|\mathcal{B}^{\dagger}\right\|_{2}^{2}\right\}\|\mathcal{E}\|_{F} .
$$

Proof Since $\mathcal{A}^{\dagger}=\mathcal{V}_{1} *_{N} \mathcal{D}_{1}^{-1} *_{N} \mathcal{U}_{1}^{H}$ and $\mathcal{B}^{\dagger}=\mathcal{T}_{1} *_{N} \mathcal{S}_{1}^{-1} *_{N} \mathcal{R}_{1}^{H}$, so

$$
\begin{aligned}
& \left\|\mathcal{B}^{\dagger}-\mathcal{A}^{\dagger}\right\|_{F}^{2}=\left\|\mathcal{T}_{1} *_{N} \mathcal{S}_{1}^{-1} *_{N} \mathcal{R}_{1}^{H}-\mathcal{V}_{1} *_{N} \mathcal{D}_{1}^{-1} *_{N} \mathcal{U}_{1}^{H}\right\|_{F}^{2} \\
& =\left\|\mathcal{T}^{H} *_{N}\left(\mathcal{T}_{1} *_{N} \mathcal{S}_{1}^{-1} *_{N} \mathcal{R}_{1}^{H}-\mathcal{V}_{1} *_{N} \mathcal{D}_{1}^{-1} *_{N} \mathcal{U}_{1}^{H}\right) *_{N} \mathcal{U}\right\|_{F}^{2} \\
& =\left\|\left[\begin{array}{c}
\mathcal{T}_{1}^{H} \\
\mathcal{T}_{2}^{H}
\end{array}\right] *_{N}\left(\mathcal{T}_{1} *_{N} \mathcal{S}_{1}^{-1} *_{N} \mathcal{R}_{1}^{H}-\mathcal{V}_{1} *_{N} \mathcal{D}_{1}^{-1} *_{N} \mathcal{U}_{1}^{H}\right) *_{N}\left[\mathcal{U}_{1} \mathcal{U}_{2}\right]\right\|_{F}^{2} \\
& =\left\|\left[\begin{array}{cc}
\mathcal{S}_{1}^{-1} *_{N} \mathcal{R}_{1}^{H} *_{N} \mathcal{U}_{1}-\mathcal{T}_{1}^{H} *_{N} \mathcal{V}_{1} *_{N} \mathcal{D}_{1}^{-1} & \mathcal{S}_{1}^{-1} *_{N} \mathcal{R}_{1}^{H} *_{N} \mathcal{U}_{2} \\
-\mathcal{T}_{2}^{H} *_{N} \mathcal{V}_{1} *_{N} \mathcal{D}_{1}^{-1} & \mathcal{O}
\end{array}\right]\right\|_{F}^{2} \\
& =\left\|\left[\begin{array}{cc}
\mathcal{S}_{1}^{-1} *_{N} \mathcal{R}_{1}^{H} *_{N} \mathcal{E} *_{N} \mathcal{V}_{1} *_{N} \mathcal{D}_{1}^{-1} & \mathcal{S}_{1}^{-2} *_{N} \mathcal{T}_{1}^{H} *_{N} \mathcal{E}^{H} *_{N} \mathcal{U}_{2} \\
\mathcal{T}_{2}^{H} *_{N} \mathcal{E}^{H} *_{N} \mathcal{U}_{1} *_{N} \mathcal{D}^{-2} & \mathcal{O}
\end{array}\right]\right\|_{F}^{2} .
\end{aligned}
$$


On using properties of Frobenius norm, we obtain

$$
\begin{aligned}
& \left\|\mathcal{B}^{\dagger}-\mathcal{A}^{\dagger}\right\|_{F}^{2} \\
& =\left\|\mathcal{S}_{1}^{-1} *_{N} \mathcal{R}_{1}^{H} *_{N} \mathcal{E} *_{N} \mathcal{V}_{1} *_{N} \mathcal{D}_{1}^{-1}\right\|_{F}^{2}+\left\|\mathcal{S}_{1}^{-2} *_{N} \mathcal{T}_{1}^{H} *_{N} \mathcal{E}^{H} *_{N} \mathcal{U}_{2}\right\|_{F}^{2}+\left\|\mathcal{T}_{2}^{H} *_{N} \mathcal{E}^{H} *_{N} \mathcal{U}_{1} *_{N} \mathcal{D}^{-2}\right\|_{F}^{2} \\
& \leq \frac{1}{\sigma_{r}^{2} \delta_{s}^{2}}\left\|\mathcal{R}_{1}^{H} *_{N} \mathcal{E} *_{N} \mathcal{V}_{1}\right\|_{F}^{2}+\frac{1}{\delta_{s}^{4}}\left\|\mathcal{T}_{1}^{H} *_{N} \mathcal{E}^{H} *_{N} \mathcal{U}_{2}\right\|_{F}^{2}+\frac{1}{\sigma_{r}^{4}}\left\|\mathcal{T}_{2}^{H} *_{N} \mathcal{E}^{H} *_{N} \mathcal{U}_{1}\right\|_{F}^{2} \\
& \leq \max \left\{\frac{1}{\sigma_{r}^{2} \delta_{s}^{2}}, \frac{1}{\delta_{s}^{4}}, \frac{1}{\sigma_{r}^{4}}\right\}\left(\left\|\mathcal{R}_{1}^{H} *_{N} \mathcal{E}_{*_{N}} \mathcal{V}_{1}\right\|_{F}^{2}+\left\|\mathcal{T}_{1}^{H} *_{N} \mathcal{E}^{H} *_{N} \mathcal{U}_{2}\right\|_{F}^{2}+\left\|\mathcal{T}_{2}^{H} *_{N} \mathcal{E}^{H} *_{N} \mathcal{U}_{1}\right\|_{F}^{2}\right) .(41)
\end{aligned}
$$

Similarly,

$$
\begin{aligned}
& \left\|\mathcal{B}^{\dagger}-\mathcal{A}^{\dagger}\right\|_{F}^{2}=\left\|\left[\begin{array}{c}
\mathcal{V}_{1}^{H} \\
\mathcal{V}_{2}^{H}
\end{array}\right] *_{N}\left(\mathcal{T}_{1} *_{N} \mathcal{S}_{1}^{-1} *_{N} \mathcal{R}_{1}^{H}-\mathcal{V}_{1} *_{N} \mathcal{D}_{1}^{-1} *_{N} \mathcal{U}_{1}^{H}\right) *_{N}\left[\begin{array}{ll}
\mathcal{R}_{1} & \mathcal{R}_{2}
\end{array}\right]\right\|_{F}^{2} \\
& =\left\|\left[\begin{array}{cc}
\mathcal{V}_{1}^{H} *_{N} \mathcal{T}_{1} *_{N} \mathcal{S}_{1}^{-1}-\mathcal{D}_{1}^{-1} *_{N} \mathcal{U}_{1}^{H} *_{N} \mathcal{R}_{1}-\mathcal{D}_{1}^{-1} *_{N} \mathcal{U}_{1}^{H} *_{N} \mathcal{R}_{2} \\
\mathcal{V}_{2}^{H} *_{N} \mathcal{T}_{1} *_{N} \mathcal{S}_{1}^{-1} & \mathcal{O}
\end{array}\right]\right\|_{F}^{2} \\
& =\left\|\left[\begin{array}{cc}
\mathcal{D}_{1}^{-1} *_{N} \mathcal{U}_{1}^{H} *_{N} \mathcal{E} *_{N} \mathcal{T}_{1} *_{N} \mathcal{S}_{1}^{-1} & \mathcal{D}_{1}^{-2} *_{N} \mathcal{V}_{1}^{H} *_{N} \mathcal{E}^{H} *_{N} \mathcal{R}_{2} \\
\mathcal{V}_{2}^{H} *_{N} \mathcal{E}^{H} *_{N} \mathcal{R}_{1} *_{N} \mathcal{S}_{1}^{-2} & \mathcal{O}
\end{array}\right]\right\|_{F}^{2} \\
& \leq \max \left\{\frac{1}{\sigma_{r}^{2} \delta_{s}^{2}}, \frac{1}{\delta_{s}^{4}}, \frac{1}{\sigma_{r}^{4}}\right\}\left(\left\|\mathcal{U}_{1}^{H} *_{N} \mathcal{E} *_{N} \mathcal{T}_{1}\right\|_{F}^{2}\right. \\
& \left.+\left\|\mathcal{V}_{1}^{H} *_{N} \mathcal{E}^{H} *_{N} \mathcal{R}_{2}\right\|_{F}^{2}+\left\|\mathcal{V}_{2}^{H} *_{N} \mathcal{E}^{H} *_{N} \mathcal{R}_{1}\right\|_{F}^{2}\right) \text {. }
\end{aligned}
$$

Thus, by adding inequalities (41) and (42), we get

$$
\begin{aligned}
2\left\|\mathcal{B}^{\dagger}-\mathcal{A}^{\dagger}\right\|_{F}^{2} \leq \max \left\{\frac{1}{\sigma_{1}^{2} \delta_{s}^{2}}, \frac{1}{\delta_{s}^{4}}, \frac{1}{\sigma_{r}^{4}}\right\}\left(\left\|\mathcal{R}_{1}^{H} *_{N} \mathcal{E}_{*_{N}} \mathcal{V}_{1}\right\|_{F}^{2}+\left\|\mathcal{T}_{1}^{H} *_{N} \mathcal{E}^{H} *_{N} \mathcal{U}_{2}\right\|_{F}^{2}\right. \\
+\left\|\mathcal{T}_{2}^{H} *_{N} \mathcal{E}^{H} *_{N} \mathcal{U}_{1}\right\|_{F}^{2} \\
\left.\quad+\left\|\mathcal{U}_{1}^{H} *_{N} \mathcal{E} *_{N} \mathcal{T}_{1}\right\|_{F}^{2}+\left\|\mathcal{V}_{1}^{H} *_{N} \mathcal{E}^{H} *_{N} \mathcal{R}_{2}\right\|_{F}^{2}+\left\|\mathcal{V}_{2}^{H} *_{N} \mathcal{E}^{H} *_{N} \mathcal{R}_{1}\right\|_{F}^{2}\right) \\
\leq 2 \max \left\{\frac{1}{\sigma_{r}^{2} \delta_{s}^{2}}, \frac{1}{\delta_{s}^{4}}, \frac{1}{\sigma_{r}^{4}}\right\}\|\mathcal{E}\|_{F}^{2},
\end{aligned}
$$

due to Lemma 3.27. Therefore,

$$
\begin{aligned}
\left\|\mathcal{B}^{\dagger}-\mathcal{A}^{\dagger}\right\|_{F} & \leq \max \left\{\frac{1}{\sigma_{r} \delta_{s}}, \frac{1}{\sigma_{r}^{2}}, \frac{1}{\delta_{s}^{2}}\right\}\|\mathcal{E}\|_{F} \\
& =\max \left\{\left\|\mathcal{A}^{\dagger}\right\|_{2}\left\|\mathcal{B}^{\dagger}\right\|_{2},\left\|\mathcal{A}^{\dagger}\right\|_{2}^{2},\left\|\mathcal{B}^{\dagger}\right\|_{2}^{2}\right\}\|\mathcal{E}\|_{F} .
\end{aligned}
$$

More on perturbation theory will appear in our next work. We would further like to bring the attention of an interested reader to the very recent work by Liu and Zhen [18], where the authors provided an algorithm to compute the spectral radius of a nonnegative tensor. This will help to find an iterative solution of a tensor multilinear system of the form $\mathcal{A} *_{N} \mathcal{X}=\mathcal{B}$ where $\mathcal{A} \in \mathbb{R}^{I_{1} \times \cdots \times I_{M} \times J_{1} \times \cdots \times J_{N}}$ and $\mathcal{B} \in \mathbb{R}^{I_{1} \times \cdots \times I_{M}}$ are given. To do this, we introduce below the definition of a sub-proper splitting of a tensor $\mathcal{A}$.

Definition 3.29 A splitting $\mathcal{A}=\mathcal{U}-\mathcal{V}$ of a tensor $\mathcal{A} \in \mathbb{C}^{I_{1} \times \cdots \times I_{M} \times J_{1} \times \cdots \times J_{N}}$ is called a sub-proper splitting if $\mathcal{U} *_{N} \mathcal{U}^{\dagger} *_{M} \mathcal{A}=\mathcal{A}$ and $\mathcal{A} *_{N} \mathcal{U}^{\dagger} *_{M} \mathcal{U}=\mathcal{A}$. 
The next result helps us to frame the iteration scheme for solving the tensor multilinear system $\mathcal{A} *_{N} \mathcal{X}=\mathcal{B}$.

Theorem 3.30 Let $\mathcal{A}=\mathcal{U}-\mathcal{V}$ be a sub-proper splitting of a tensor $\mathcal{A} \in \mathbb{C}^{I_{1} \times \cdots \times I_{M} \times J_{1} \times \cdots \times J_{N}}$. Then $\mathcal{A}=\mathcal{U} *_{N}\left(\mathcal{I}-\mathcal{U}^{\dagger} *_{M} \mathcal{V}\right)$. Further, if $1 \notin \Omega\left(\mathcal{U}^{\dagger} *_{M} \mathcal{V}\right)$, then

1. $\mathcal{A}^{\dagger}=\left(\mathcal{I}-\mathcal{U}^{\dagger} *_{M} \mathcal{V}\right)^{-1} *_{N} \mathcal{U}^{\dagger}$;

2. If the system $\mathcal{A} *_{N} \mathcal{X}=\mathcal{B}$ is consistent, then every solution $\mathcal{X}$ satisfying $\mathcal{U}^{\dagger} *_{M} \mathcal{U} *_{N} \mathcal{X}=\mathcal{X}$ (of which at least one exists, e.g., $\mathcal{A}^{\dagger} *_{M} \mathcal{B}$ ) satisfies $\mathcal{X}=\mathcal{U}^{\dagger} *_{M} \mathcal{V} *_{N} \mathcal{X}+\mathcal{U}^{\dagger} *_{M} \mathcal{B}$.

Proof For a sub-proper splitting $\mathcal{A}=\mathcal{U}-\mathcal{V}$ of a tensor $\mathcal{A} \in \mathbb{C}^{I_{1} \times \cdots \times I_{M} \times J_{1} \times \cdots \times J_{N}}$, we have $\mathcal{U} *_{N} \mathcal{U}^{\dagger} *_{M} \mathcal{V}=\mathcal{V}$ and $\mathcal{V} *_{N} \mathcal{U}^{\dagger} *_{M} \mathcal{U}=\mathcal{V}$. Thus, we obtain $\mathcal{A}=\mathcal{U} *_{N}\left(\mathcal{I}-\mathcal{U}{ }^{\dagger} *_{M} \mathcal{V}\right)$

The first case of the second part follows immediately from Theorem 3.22. Also, let $\mathcal{X}_{0}$ be a solution of the consistent multilinear system $\mathcal{A} *_{N} \mathcal{X}=\mathcal{B}$ which satisfies $\mathcal{U}{ }^{\dagger} *_{M} \mathcal{U} *_{N} \mathcal{X}_{0}=\mathcal{X}_{0}$. Thus, $\mathcal{U} *_{N} \mathcal{X}_{0}=\mathcal{V} *_{N} \mathcal{X}_{0}+\mathcal{B}$. Now, by pre-multiplying $\mathcal{U}^{\dagger}$, we obtain $\mathcal{X}_{0}=\mathcal{U}^{\dagger} *_{M} \mathcal{V} *_{N} \mathcal{X}_{0}+$ $\mathcal{U}^{\dagger} *_{M} \mathcal{B}$.

Therefore, the iteration scheme

$$
\mathcal{X}_{k+1}=\mathcal{U}^{\dagger} *_{M} \mathcal{V}_{*_{N}} \mathcal{X}_{k}+\mathcal{U}^{\dagger} *_{M} \mathcal{B}
$$

will converge to $\mathcal{A}^{\dagger} *_{M} \mathcal{B}$, the least-squares solution of minimum norm of the tensor multilinear system $\mathcal{A} *_{N} \mathcal{X}=\mathcal{B}$ if the spectral radius of $\mathcal{U}^{\dagger} *_{M} \mathcal{V}$ is less than 1.

\section{Concluding remarks}

In this paper, we have studied the problem of the reverse-order law for arbitrary order tensors. The advantage of this study is that the proofs do not use the notion of the range and the null space of a tensor. The important findings are summarized as follows:

- The problem of the reverse-order law for arbitrary order tensors is taken up in Sect. 3.1.

- Some new properties of a $\{1,2,3\}$-inverse and a $\{1,2,4\}$-inverse of a tensor are examined next in Sect. 3.1.1.

- As an application of the reverse-order law, the problem of computing the additive MoorePenrose inverse of tensors is partially attempted in Sect. 3.2. The general case is left for future study and is posed as an open problem below.

- Problem: How to compute the Moore-Penrose inverse of the sum of two arbitrary order tensors $\mathcal{A}$ and $\mathcal{B}$ ?

- The notion of the Frobenius norm and the spectral norm for arbitrary order tensors are then recalled to analyze the additive perturbation bounds for the Moore-Penrose inverse of arbitrary order tensors via the Einstein product by means of Frobenius norm. More on additive perturbation bounds using the spectral norm and the $Q$-norm will appear in our next article.

- Finally, we have introduced the notion of the sub-proper splitting of a tensor to find an iterative solution of a tensor multilinear system. The detailed convergence analysis of the iteration scheme (43) can be further analyzed by the help of the reverse-order law, and is left for a future topic of research interest.

Acknowledgements We thank the Government of India for introducing the work from home initiative during the COVID-19 pandemic. This research was supported by grant CRG/2018/002986 from the Science and Engineering Research Board, Department of Science and Technology, New Delhi, India. 


\section{References}

1. Behera, R., Mishra, D.: Further results on generalized inverses of tensors via the Einstein product. Linear Multilinear Algebra 65, 1662-1682 (2017)

2. Beylkin, G., Mohlenkamp, M.J.: Algorithms for numerical analysis in high dimensions. SIAM J. Sci. Comput. 26, 2133-2159 (2005)

3. Beylkin, G., Mohlenkamp, M.J.: Numerical operator calculus in higher dimensions. Proc. Natl. Acad. Sci. USA 99, 10246-10251 (2002)

4. Brazell, M., Li, N., Navasca, C., Tamon, C.: Solving multilinear systems via tensor inversion. SIAM J. Matrix Anal. Appl. 34, 542-570 (2013)

5. Che, M., Wei, Y.: Theory and Computation of Complex Tensors and its Applications. Springer, Singapore (2020)

6. Einstein, A.: The foundation of the general theory of relativity. In: Kox, A.J., Klein, M.J., Schulmann, R. (eds.) The collected papers of Albert Einstein 6, pp. 146-200. Princeton University Press, Princeton (2007)

7. Hackbusch, W., Khoromskij, B.N.: Tensor-product approximation to operators and functions in high dimensions. J. Complex 23, 697-714 (2007)

8. Hackbusch, W., Khoromskij, B.N., Tyrtyshnikov, E.E.: Hierarchical kronecker tensor product approximations. J. Numer. Math. 13, 119-156 (2005)

9. Huang, S., Zhao, G., Chen, M.: Tensor extreme learning design via generalized Moore-Penrose inverse and triangular type-2 fuzzy sets. Neural Comput. Appl. 31, 5641-5651 (2019)

10. Ji, J., Wei, Y.: The Drazin inverse of an even-order tensor and its application to singular tensor equations. Comput. Math. Appl. 75, 3402-3413 (2018)

11. Ji, J., Wei, Y.: Weighted Moore-Penrose inverses and fundamental theorem of even-order tensors with Einstein product. Front. Math. China 12, 1319-1337 (2017)

12. Jin, H., Bai, M., Benítez, J., Liu, X.: The generalized inverses of tensors and an application to linear models. Comput. Math. Appl. 74, 385-397 (2017)

13. Khoromskij, B.N., Khoromskaia, V., Flad, H.-J.: Numerical solution of the Hatree-Fock equation in multilevel tensor-structured format. SIAM J. Sci. Comput. 33, 45-65 (2011)

14. Khoromskij, B.N.: Tensor-structured numerical methods in scientific computing: Survey on recent advances. Chemom. Intell. Lab. Syst. 110, 1-19 (2012)

15. Kolda, T., Bader, B.W.: Tensor decompositions and applications. SIAM Rev. 51, 455-500 (2009)

16. Lai, W.M., Rubin, D., Krempl, E.: Introduction to Continuum Mechanics. Butterworth-Heinemann, Oxford (2009)

17. Lathauwer, L.D.: A Survey of Tensor Methods. ISCAS, Taipei (2009)

18. Liu, Q., Zhen, C.: An algorithm for computing the spectral radius of nonnegative tensors. Comput. Appl. Math. 38 (2019) Article number: 90

19. Liang, M., Zheng, B.: Further results on Moore-Penrose inverses of tensors with application to tensor nearness problems. Comput. Math. Appl. 77, 1282-1293 (2019)

20. Ma, H., Li, N., Stanimorivić, P.S., Katsikis, V.N.: Perturbation theory for Moore-Penrose inverse of tensor via Einstein product. Comput. Appl. Math. 38, 111 (2019)

21. Panigrahy, K., Behera, R., Mishra, D.: Reverse-order law for the Moore-Penrose inverses of tensors. Linear Multilinear Algebra 68, 246-264 (2020)

22. Panigrahy, K., Mishra, D.: Extension of Moore-Penrose Inverse of Tensor via Einstein product. Linear Multilinear Algebra (2020). https://doi.org/10.1080/03081087.2020.1748848

23. Qi, L., Luo, Z.: Tensor Analysis: Spectral Theory and Special Tensors. SIAM, Philadelphia (2017)

24. Smilde, A., Bro, R., Geladi, P.: Multi-Way Analysis: Applications in the Chemical Sciences. Wiley, West Sussex (2004)

25. Stanimirović, P.S., Ćirić, M., Katsikis, V.N., Li, C., Ma, H.: Outer and (b, c) inverses of tensors. Linear Multilinear Algebra. 68, 940-971 (2020)

26. Sun, L., Zheng, B., Bu, C., Wei, Y.: Moore-Penrose inverse of tensors via Einstein product. Linear Multilinear Algebra. 64, 686-698 (2016)

27. Sun, L., Zheng, B., Wei, Y., Bu, C.: Generalized inverses of tensors via a general product of tensors. Front. Math. China. 13, 893-911 (2018)

28. Tewarson, R.P., Narain, P.: Solution of linear equations resulting from satellite remote soundings. J. Math. Anal. Appl. 47, 1-14 (1974)

29. Wang, G., Wei, Y., Qiao, S.: Generalized Inverses: Theory and Computations. Second edition. Developments in Mathematics, 53. Springer, Singapore; Science Press Beijing, Beijing (2018)

30. Wei, Y., Ding, W.: Theory and Computation of Tensors: Multi-Dimensional Arrays. Elsevier, Amsterdam (2016) 
31. Wei, Y., Stanimirović, P., Petković, M.: Numerical and Symbolic Computations of Generalized Inverses. World Scientific Publishing Co.Pte. Ltd., Hackensack (2018)

Publisher's Note Springer Nature remains neutral with regard to jurisdictional claims in published maps and institutional affiliations. 